\title{
Experimental and theoretical study of swept-wing boundary-layer instabilities. Unsteady crossflow instability
}

Cite as: Phys. Fluids 31, 064101 (2019); https://doi.org/10.1063/1.5094609

Submitted: 04 March 2019 . Accepted: 01 May 2019 . Published Online: 11 June 2019

V. I. Borodulin, A. V. Ivanov, Y. S. Kachanov (D, D. A. Mischenko, R. Örlü (D), A. Hanifi, and S. Hein

\section{CAPTURE WHAT'S POSSIBLE}

\section{WITH OUR NEW PUBLISHING ACADEMY RESOURCES}

Learn more $\Theta$

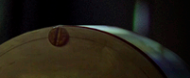




\title{
Experimental and theoretical study of swept-wing boundary-layer instabilities. Unsteady crossflow instability
}

\author{
Cite as: Phys. Fluids 31, 064101 (2019); doi: 10.1063/1.5094609 \\ Submitted: 4 March 2019 - Accepted: 1 May 2019 • \\ Published Online: 11 June 2019
}

\begin{abstract}
V. I. Borodulin, ${ }^{1}$ A. V. Ivanov, ${ }^{1}$ Y. S. Kachanov, ${ }^{1, a)}$ (D) D. A. Mischenko, ${ }^{1}$ R. Örlü, ${ }^{2}$ (D) A. Hanifi, ${ }^{2}$ and S. Hein
AFFILIATIONS

${ }^{1}$ Khristianovich Institute of Theoretical and Applied Mechanics, Novosibirsk 630090, Russian Federation

${ }^{2}$ Department of Mechanics, Linné FLOW Centre, KTH Royal Institute of Technology, SE-100 44 Stockholm, Sweden

${ }^{3}$ Institute of Aerodynamics and Flow Technology, DLR, 37073 Göttingen, Germany
\end{abstract}

a) E-mail: kachanov@itam.nsc.ru

\begin{abstract}
Extensive combined experimental and theoretical investigations of the linear evolution of unsteady (in general) Cross-Flow (CF) and threedimensional (3D) Tollmien-Schlichting (TS) instability modes of 3D boundary layers developing on a swept airfoil section have been carried out. CF-instability characteristics are investigated in detail at an angle of attack of $-5^{\circ}$ when this kind of instability dominates in the laminarturbulent transition process, while the 3D TS-instability characteristics are studied at an angle of attack of $+1.5^{\circ}$ when this kind of instability is predominant in the transition process. All experimental results are deeply processed and compared with results of calculations based on several theoretical approaches. For the first time, very good quantitative agreement of all measured and calculated stability characteristics of swept-wing boundary layers is achieved both for unsteady CF- and 3D TS-instability modes for the case of a boundary layer developing on a real swept airfoil. The first part of the present study (this paper) is devoted to the description of the case of CF-dominated transition, while the TS-dominated case will be described in detail in a subsequent second part of this investigation.
\end{abstract}

Published under license by AIP Publishing. https://doi.org/10.1063/1.5094609

\section{INTRODUCTION}

Laminar-turbulent transition in three-dimensional (3D) boundary-layer flows is of great scientific interest and significant importance for a wide range of technical applications. Its importance for aeronautical application has increased with the need for low-emission aircraft.

In general, there are several flow instability mechanisms that trigger transition in the swept-wing boundary layers, each having their own characteristics as well as sources of their excitation. The most significant of these instability mechanisms are (a) the attachment-line instability, ${ }^{1}$ (b) the cross-flow (CF) instability (see, e.g., Refs. 2 and 3), (c) the Tollmien-Schlichting (TS) instability (see, e.g., Ref. 4), and (d) the instability of separated shear layers that is important in presence of laminar separation zones (see, e.g., Ref. 5).

The relative contribution of these modes to the transition process depends on the pressure gradient and the sweep angle.
For instance, in case of a favorable, i.e., negative, streamwise pressure gradient (such as on the upper side of a long-laminar-run swept wing installed at a small positive or negative angle of attack) and a sufficiently large sweep angle, one may expect predominance of the stationary and/or traveling CF-instability modes inducing very specific transition scenarios (see, e.g., Ref. 6) whereas, in the case of nearly zero chordwise pressure gradients or weak adverse ones, the TS-instability (oblique) waves may prevail (see, e.g., Ref. 4). Although the CF-mode instability of swept-wing boundary layers has been studied intensively for many years (see, e.g., Refs. 7-11 for an overview), the detailed qualitative agreement between all measured and calculated linear stability characteristics has not been obtained yet for real nonparallel boundary layers developing on curved surfaces of real swept airfoils. This is especially true for nonstationary CF-instability modes. For the 3D TSinstability characteristics of swept-wing boundary layers, the investigations are even more rare. This can be explained by the extreme 
complexity of accurate quantitative experimental investigations of these problems.

Initially, the majority of experimental investigations of the cross-flow instability were devoted basically to stationary disturbances-the so-called cross-flow vortices (see, e.g., pioneering works in Refs. 12-23 and more recent studies, e.g., Refs. 24 and 25). These modes often prevail in the spectrum under the socalled "natural" (i.e., uncontrolled) disturbance conditions when the free-stream turbulence level is not very high. This can be explained by the fact that the stationary cross-flow modes are generated near the swept-wing leading edge by microscopically small roughness (or waviness) of the surface that can be rather large as compared to a small boundary-layer thickness. ${ }^{26}$ At the same time, calculations (see, e.g., Refs. 3, 15, and 27-29) predict that the swept-wing boundary layer is the most unstable to the traveling waves, i.e., to the unsteady cross-flow instability modes. The traveling modes are also observed experimentally under "natural" conditions, but usually when the free-stream turbulence is relatively high and/or the surface is relatively smooth. ${ }^{16,30,31}$ For a general characterization of the transition process initiated by the CF instability in various practically important cases, both steady and traveling modes must be considered.

Early experiments with stationary CF modes performed under "natural" disturbance conditions showed qualitative agreement of characteristics of the observed disturbances with general properties of the CF vortices predicted by the linear stability theory, although quantitative values of the growth rates of the most amplified CF modes predicted numerically were found to be much higher than those observed in uncontrolled experiments for the most intensive boundary-layer disturbances. ${ }^{20,26}$

Experimental results under controlled disturbance conditions have demonstrated, in contrast, rather good agreement with the linear stability theory for the cross-flow vortices. For the first time, quantitative values of the stability characteristics of a swept-wing boundary layer with respect to normal (i.e., harmonic in space) cross-flow vortices were obtained experimentally in Refs. 17, 19, and 32 employing various methods of excitation of CF vortices and a procedure of complete Fourier decomposition of stationary disturbances into the normal oblique modes. The measurements were performed on a swept-plate experimental model with a chordwise pressure gradient initiated by a contoured wall bump. It was found that the linear stability theory (calculations by Fedorov) predicts very well the growth rates and other characteristics of the sweptwing flow stability to the cross-flow vortices. Later experiments in Ref. 33, performed on a real swept airfoil with a curved surface, have also shown a very good agreement with calculations ${ }^{34}$ based on the parabolized stability equations (PSE). Those experiments considered the excitation of a small set of discrete spanwise-wavenumber modes. An important role of the surface curvature was shown.

Observations of traveling instability modes in the swept-wing boundary layer were made in experiments by Refs. 16, 35-39, and others. These experiments (except for Ref. 36) were performed under "natural" disturbance conditions when the initial spectrum of perturbations was uncontrolled. Such experiments are very useful for observation of some typical properties of traveling waves in the $3 \mathrm{D}$ boundary-layer transition, which were documented. However, with the "natural" disturbances, it is only possible to obtain information about the development of the most intensive fluctuations rather than those of the most amplified CF modes. A comparison of the traveling-wave growth rates observed experimentally with those predicted for the most amplified modes by the linear stability theory showed their significant disagreement. ${ }^{20,40}$ First, very good agreement of all measured and calculated CF-instability characteristics for traveling (and also stationary) normal modes was obtained in Refs. 41 and 42 for the case of a $25^{\circ}$ swept-wing model having a flat surface.

Most of the theoretical work on the 3D boundary-layer stability was focused initially on convective instabilities subject to the parallel-flow approximation (see, e.g., Ref. 27). Later, a number of more advanced approaches were developed, in particular, the parabolized stability equations (PSEs). Nonlinear PSE calculations can capture some of the features observed in experiments (e.g., Refs. 34 and 43-46). Direct numerical simulations have also been used to investigate different aspects of the CF instability and transition (see, e.g., Refs. 11 and 47-53). However, detailed comparisons between theory and experiments remain limited, especially for traveling cross-flow modes and are unavailable for the cases of real swept airfoils simulating infinite-span swept wings with surface curvature.

The transition location in the CF-dominated boundary-layer flows on swept wings depends on four main aspects: (i) the spectra of the flow and surface perturbations, (ii) the boundary-layer receptivity characteristics, (iii) the steady and unsteady CF-instability characteristics, and (iv) the leading mechanisms of nonlinear disturbance interactions.

The evolution of the instability waves depends significantly on particular base-flow and disturbance conditions such as model geometry, mean-flow parameters, and environmental disturbance parameters. That is why an accurate analysis of the receptivity problem requires precise measurements of both stability and receptivity characteristics to be carried out promptly, within one wind tunnel session, when the atmospheric parameters are nearly constant. The development of the appropriate experimental procedure for the receptivity and stability measurements was the first goal of the present study.

This work was a part of the European project RECEPT. The project was devoted basically to the investigation of the problem of receptivity of swept-wing boundary layers to various external perturbations, such as surface nonuniformities and free-stream vortices. However, detailed examination of various stability characteristics of the boundary layers under study is also an important prerequisite aspect of the receptivity investigation. In the present paper (first part of the study), we focus on the characteristics of the unsteady CF-instability modes in an accelerating boundary layer over a swept wing. Here, we report detailed experimental results and quantitative comparison with the theory based on the local and nonlocal (PSE) approaches. Some results of the present investigation were discussed as a preliminary in the first section of a short publication in conference proceedings, ${ }^{54}$ while their complete analysis is given below.

\section{EXPERIMENTAL PROCEDURE AND THEORETICAL APPROACHES}

\section{A. Experimental setup}

The measurements were performed in the Minimum Turbulence Level (MTL) wind tunnel of the Royal Institute of Technology 
(Stockholm) at a free-stream speed of about $11 \mathrm{~m} / \mathrm{s}$. A section of a $35^{\circ}$ swept-wing airfoil with a chord length of $800 \mathrm{~mm}$ was mounted in the wind-tunnel test section at a $-5^{\circ}$ angle of attack. In order to provide the spanwise base-flow uniformity (to meet the infiniteswept wing condition), the model was equipped with contoured sidewalls (see Refs. 55-57). Their shapes were calculated numerically based on the infinite-swept wing assumption and corresponded to that of the potential flow at the boundary-layer edge. ${ }^{55} \mathrm{~A}$ general view on the model in the wind-tunnel test section is given in Fig. 1(a).

The coordinates used in the present paper are the following. The $x$-axis is directed parallel to the incident flow velocity vector $C_{o}$, along the test section centerline. The $z$-axis is normal to the $x$-axis [see Fig. 1(a)]. Point $(x, z)=(0,0)$ is located at the middle of the airfoil section leading edge. Another coordinate system used is $\left(x^{\prime}, z^{\prime}\right)$. It was obtained by two subsequent rotations of the $(x, z)$ system. First, both the $x$ - and $z$-axes were turned by $35^{\circ}$ (sweep angle) around the axis normal to the $(x, z)$-plane passing through the point $(0,0)$. The next turn was made around the leading edge, by $-5^{\circ}$ (angle of attack). As a result, the $z^{\prime}$-axis coincides with the leading edge and the chord line lies on the $x^{\prime}$-axis. The value $x^{\prime} / c=1$ corresponds to the trailing edge of the experimental model $(c=800 \mathrm{~mm})$. One more important streamwise coordinate is $x_{s}^{\prime}$. It is a curvilinear one. It starts at the model leading edge and goes in the chordwise direction (similar to the $x^{\prime}$-axis) but along the model surface. One more $\left(x^{*}, z^{*}\right)$-coordinate system used in the present paper is a local one with the $x^{*}$-axis directed along the mean velocity vector $C_{e}$ of the potential flow near the external edge of the boundary layer. (In the experiments, the orientation of the vector $C_{e}$ was measured at $y=10 \mathrm{~mm}$ based on the wake-wire technique described below.) It is also convenient to use an additional axis $z_{c}^{\prime}$, which is like the $z^{\prime}$-axis but at all chordwise locations have their origin at the centerline of the experimental model, i.e., at the $x$-axis (with $z=0$ ). The $y$-coordinate is also a local one; it is normal to the model surface, and its origin $y=0$ lies always on the airfoil section surface.

The experiments were performed at fully controlled disturbance conditions. The velocity perturbations were excited in the airfoil boundary layer by means of a special disturbance source located at chordwise position $x^{\prime} / c=0.15\left(x^{\prime}=120 \mathrm{~mm}, x_{s}^{\prime}=131.8 \mathrm{~mm}\right)$. The main part of the source represented a spanwise row of circular latex membranes, mounted flush with the model surface oscillating under the influence of pressure fluctuations excited by a set of speakers, playing a role of pumps [Fig. 1(b)]. The speakers were located outside the wind-tunnel test section and were connected to the source by an array of plastic pipes. The spanwise spacing of the membranes was selected to be equal to $8 \mathrm{~mm}$ in the main measurements. In various regimes of measurements, either all membranes or part of them (including the important case of one membrane) were activated. At the spanwise-periodic excitation, the spanwise wavelength $\lambda_{z}^{\prime}$ of the surface vibrations was equal to $16 \mathrm{~mm}$. Depending of the level of excitation, the membranes oscillated with amplitudes from some tenths of a micron to several dozens of microns.

Velocity fields in the potential flow and inside the boundary layer were examined by means of a hot-wire anemometer. In order to obtain the streamwise and spanwise potential-flow velocity components experimentally, which are required for exactly matching the actual flow characteristics with those predicted theoretically, the standard double-wire hot-wire measurements were performed in the potential flow (see Ref. 56). The results of these measurements provided important information about compliance with the infiniteswept wing condition at large distances from the surface, as well as about blockage effects produced by the main MTL traverse. However, these measurements were carried out at rather large distances from the airfoil surface. Therefore, additional two-component measurements were necessary in the vicinity of the boundary-layer edge. They were performed by means of an original wake-wire technique combined with measurements by single-wire probes performed both at the boundary-layer edge and inside the boundary layer. This technique is described in Ref. 54. Subsequent processing of the wake-wire and single hot-wire measurements gave us potentialflow streamlines in a rather extended streamwise range of about $400 \mathrm{~mm}$ covering the whole region of interest. These kinds of data also enabled performing separation of the velocity components and finding, in particular, the spanwise components of the potential flow velocity vectors. The results of such measurements are presented in Sec. II D. Some details related to the experimental setup can be also found in Refs. 55-57.

\section{B. Procedure of CF-instability measurements}

The CF-instability measurements were carried out at fully controlled disturbance conditions with the excitation of boundarylayer perturbations by the surface disturbance source mentioned above. All excited disturbances were harmonic in time (with frequency variation) and had various spanwise-wavenumber spectra.
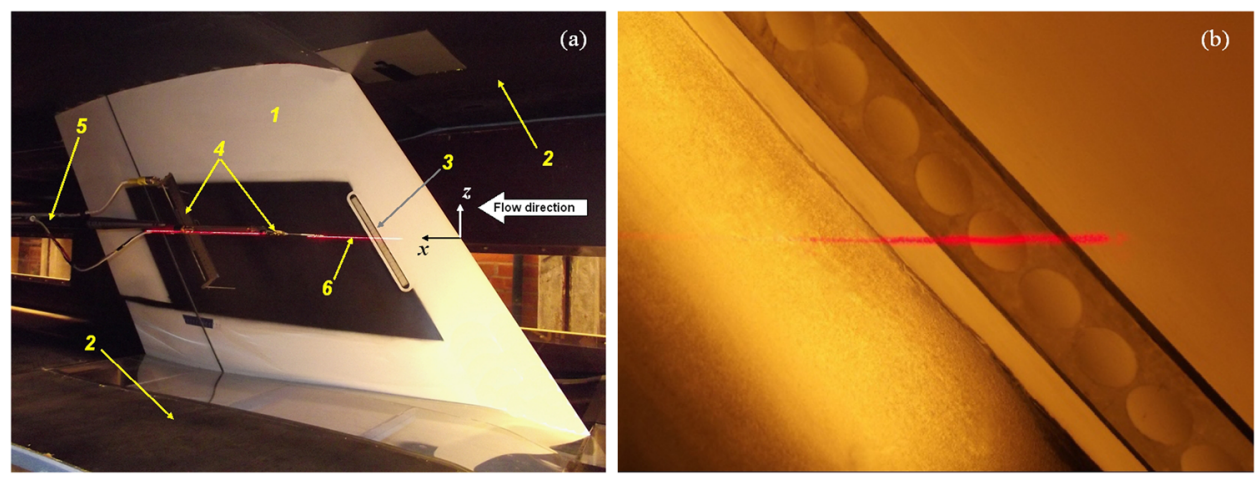

FIG. 1. (a) Experimental model of a $35^{\circ}$ swept wing installed in wind-tunnel test section. (1) Airfoil section, (2) contoured sidewalls, (3) surface disturbance source, (4) traverse Komarik, (5) sting of standard wind-tunnel traverse, and (6) laser sheet. (b) Instantaneous picture of the disturbance-source membranes deflected during oscillations at excitations of spanwise periodic disturbance (with period $\lambda_{z}^{\prime}=32 \mathrm{~mm}$ in this particular case). 
In different types of measurements, the source was used in two, basically, different ways: either with the actuation of only one surface membrane (producing spanwise-localized disturbance) or with actuation of the whole row of surface membranes (producing spanwise-periodic disturbance). In the former case, the spanwisewavenumber spectrum of the excited boundary-layer disturbances consisted of a broad range of spanwise wavenumbers but the accuracy of measurements was relatively low due to distribution of the disturbance energy among numerous modes of the frequencywavenumber spectrum. In the latter case, the spanwise-wavenumber spectrum of the excited disturbances consisted of two or three oblique (in general) modes only, but the accuracy of measurements was much higher due to concentration of the disturbance energy in this small amount of modes. Of course, we tried to avoid too high levels of excitation in order to eliminate any significant nonlinearity in the disturbance development.

The stability experiments with spanwise-periodic disturbances were basically performed in order to measure wall-normal profiles of the disturbance amplitudes and phases, for their comparison with eigenfunctions of the CF-instability modes, and for identification of, in this way, the physical nature of the boundary-layer perturbations. Additionally, these measurements were used for determining the wall-normal distances of the disturbance amplitude maxima and for the selection of some optimal wall distances (amplitude maximum location) used later in the main instability measurements conducted in cases of spanwise-localized excitation.

The stability measurements carried out for the spanwiselocalized disturbances were the main ones. Sets of spanwise distributions obtained at various chordwise coordinates for every disturbance frequency were subjected further to the spanwise Fourier decomposition for obtaining the spanwise-wavenumber spectra of the boundary-layer disturbances, as well as the streamwise wavenumbers and propagation angles for every particular frequency and spanwise wavenumber. The procedure of such complete decomposition of wave trains into fans of oblique waves was developed and applied for the first time for TS-waves propagating in the Blasius boundary layer (see details in Ref. 58). Later, similar procedures were applied to other instability modes in various boundary-layer flows including the CF-instability waves developing in a swept-wing boundary layer (see, e.g., Ref. 59) and unsteady Görtler vortices on concave walls. ${ }^{60}$

In addition, special measurements were performed for checking the linearity of the instability mechanisms under study at the particular experimental conditions (frequencies, spanwise wavenumbers, amplitudes, etc.).

\section{Theoretical approaches to base-flow and stability calculations}

\section{Base-flow calculations}

The mean flow used for stability analyses presented here is obtained through the boundary-layer computations using the pressure distribution along the model surface. In the design stage of the experiments, computational fluid dynamics (CFD) computations were performed in order to find the contours of the wind tunnel walls that ensured a spanwise-invariant flow over the surface of the model. However, the experimental setup was slightly different from that in simulations and therefore a mismatch between the computed and measured pressure distribution was observed. The differences are thought to be an effect of a slightly different angle of attack and the presence of the traverse system when stability measurements were performed. In order to get a perfect fit between the boundarylayer characteristics from the computations and the experimental data, some modifications to the computed wall-pressured distribution were made. The effective sweep angle was found to be close to $34^{\circ}$ which then was used here. The boundary-layer computations were performed based on the infinite-swept wing assumption. The comparison between the measured and the computed characteristics of the boundary layer is given in Sec. II D.

\section{Stability analysis}

The base-flow stability characteristics to the perturbations were computed using two approaches: (i) a locally parallel one and (ii) a nonlocal-nonparallel approach based on the parabolized stability equations (PSEs). The analyses are performed using the NOLOT code. $^{61,62}$ The PSE computations include effects of surface curvature, while in local stability analysis the curvature effects have been excluded. Furthermore, to mimic the experiment as closely as possible, the amplitude of the perturbations was evaluated at the same wall-normal position as in the experiment.

\section{Base-flow characteristics}

\section{Potential flow structure}

The shape of the outer streamlines was measured near the boundary-layer edge by means of the wake-wire technique mentioned above (see also Ref. 59). The measurements are performed at wall-normal distance of $10 \mathrm{~mm}$, which is very close to the boundarylayer edge but is slightly outside of it for all studied chordwise locations. The results are presented in Fig. 2(a) for three spanwise positions along with the corresponding average shape. It is seen that the orientations of streamlines are practically the same at all studied spanwise coordinates (which correspond to the spanwise range of the surface disturbance source). This result confirms the spanwise uniformity of the base flow and validity of the infinite swept-wing condition in the main region of stability and receptivity measurements.

The main results separating the potential-flow mean-velocity vector into its two surface-parallel components are presented in Fig. 2(b). As it shown there, the measured and calculated chordwise distributions of the chordwise $U_{e s^{-}}^{\prime}$ and spanwise $W_{e}^{\prime}$-components of the potential-flow velocity vector are in a close agreement with each other. It is also seen that the sweep condition $W_{e}^{\prime}=$ constant is satisfied very well in both the experiment and the Navier-Stokes computations. The acceleration of both the chordwise and streamwise velocity components provides good conditions for formation of the crossflow and for predominance of the crossflow instability. Simultaneously, the TS-instability is suppressed in this case by the favorable streamwise pressure gradient.

\section{Boundary-layer flow}

The measured and calculated wall-normal profiles of the streamwise component of the mean-velocity vector are illustrated in Fig. 3 for three chordwise positions and three spanwise positions. These locations cover the region of main stability 

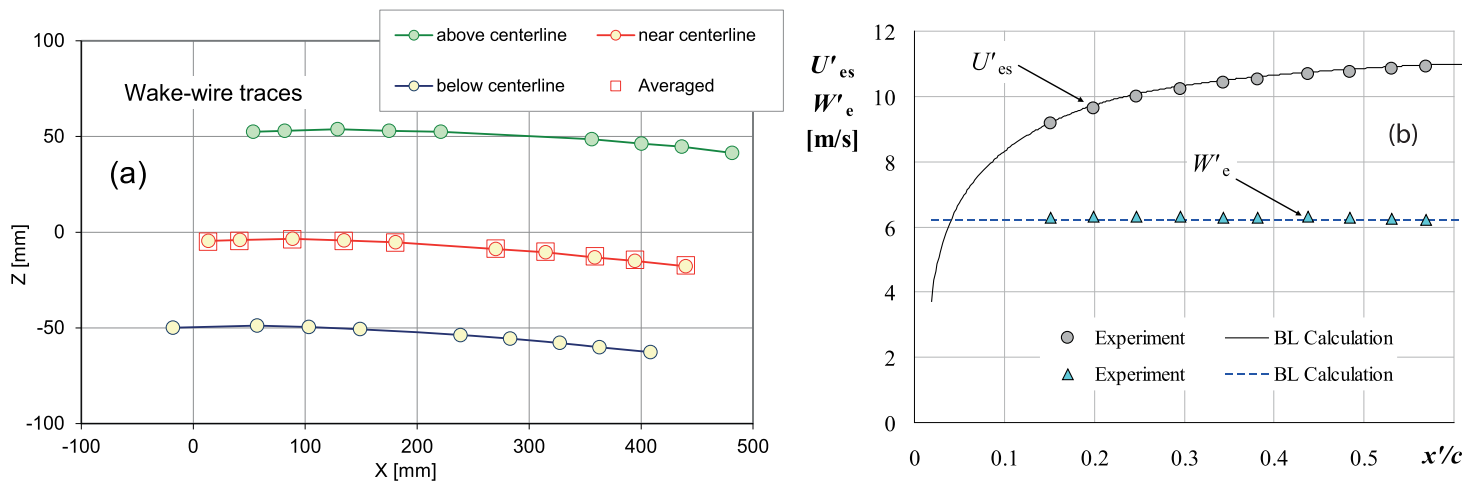

FIG. 2. (a) Streamlines of potential flow over airfoil surface measured at $y=10 \mathrm{~mm}$ at three spanwise locations and the averaged streamline. (b) Chordwise distributions of the corresponding chordwise $U_{e s}^{\prime}$ and spanwise $W_{e}^{\prime}$ velocity components.

measurements. First, it is seen that the experimental and theoretical profiles agree very well with each other. Second, it is found that the experimental data do not display any spanwise dependence of their shape, thus demonstrating the spanwise uniformity also of the viscous flow inside the boundary layer. This result is valid for the region of main measurements performed in the CF-instability experiments.

The experimental values of the boundary-layer displacement thickness $\delta_{1}$ and the shape factor $H=\delta_{1} / \delta_{2}$ (here, $\delta_{2}$ is the boundary-layer momentum thickness) were found by means of integration of the profiles like those shown in Fig. 3. They are presented in Fig. 4 in comparison with the calculated ones. It is seen that the shape factor increases slightly downstream. A very good agreement between the measured and calculated results is observed as well.

\section{E. Regimes of CF-instability measurements}

During the stability measurements, the unsteady CF modes (including quasisteady ones) were generated by the surface disturbance source described above. The disturbances called quasisteady ones have so low frequency that the flow passes several times through the whole region of measurements during one period of such mode oscillations and their stability characteristics are the same practically as those of the exactly steady CF modes. In the present experiments, these are modes with frequency of $2 \mathrm{~Hz}$. One has to note also that the accuracy of measurements at excitation of quasisteady disturbances is very much higher compared to that of measurements performed at exactly steady perturbations. In the case of spanwise-localized excitation of wave trains of CFinstability modes, the spanwise wavenumber spectrum of the modes was broadband, while in the former case, only two or three modes (with spanwise wavenumbers $\pm \beta_{1}^{\prime}$ and, sometimes, $\beta^{\prime}=0$ ) were excited.

Ranges of the studied disturbance frequencies and spanwise wavenumbers were selected based on preliminary linear-stability calculations at a stage of the experimental model design. The membranes of the surface disturbance source had a spanwise spacing of $\lambda_{z}^{\prime}=8 \mathrm{~mm}$ corresponding to maximum spanwise wavenumbers for the spanwise-periodically excited CF-instability modes of $\beta_{\max }= \pm 2 \pi / \lambda_{z}^{\prime}= \pm 0.785 \mathrm{rad} / \mathrm{mm}$. Of course, in the case of localized excitation, i.e., with only one membrane actuated, the spanwise-wavenumber range of the excited CF modes was much broader.
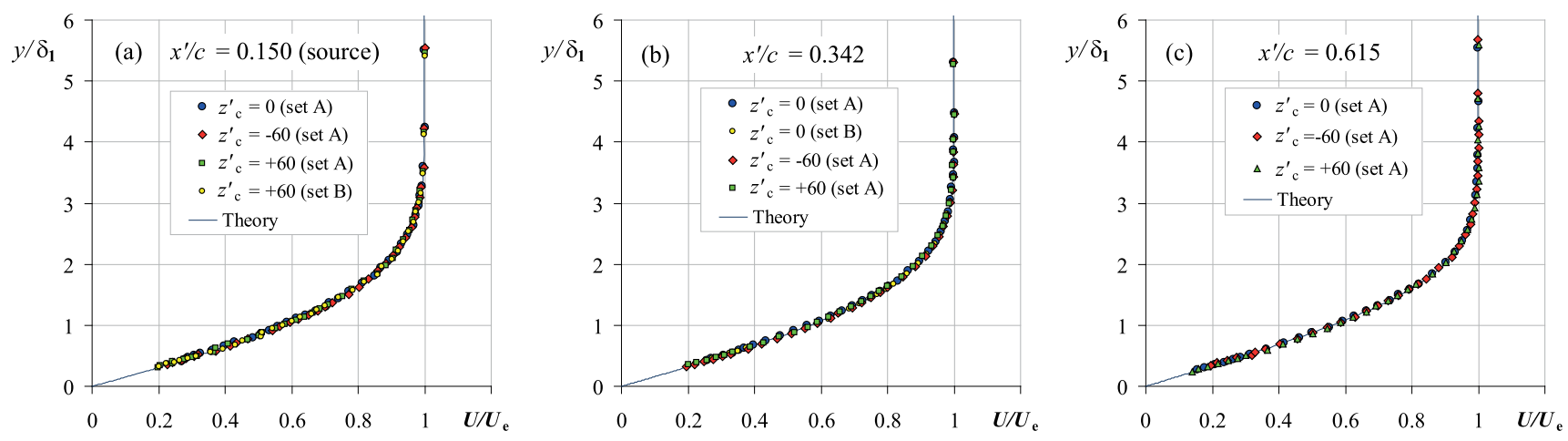

FIG. 3. Wall-normal profiles of streamwise mean-velocity component measured at three chordwise and three spanwise locations in comparison with the calculated ones. (a): $x^{\prime} / c=0.150$ (source position), (b): $x^{\prime} / c=0.342$, and (c): $x^{\prime} / c=0.615$. 


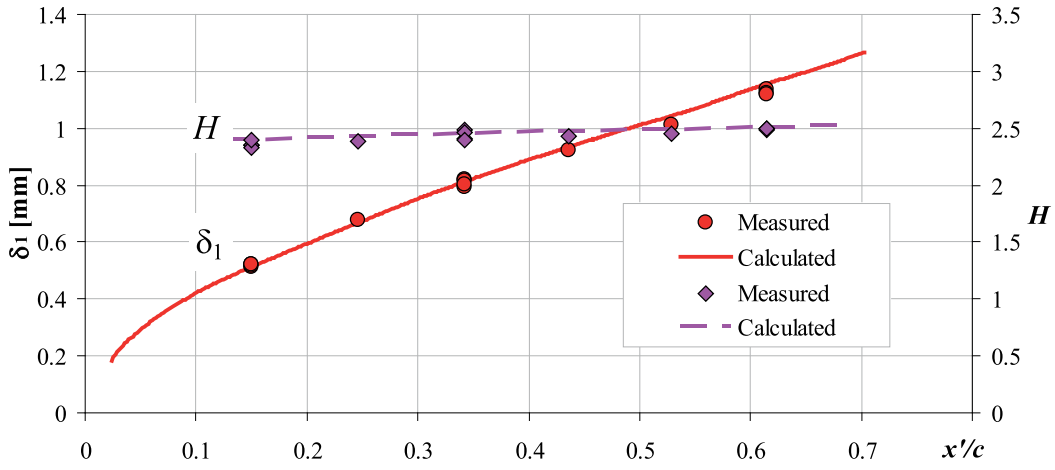

FIG. 4. Boundary-layer displacement thickness and shape factor obtained for the streamwise mean-velocity component vs chordwise coordinate $x^{\prime} / c$ from experiment in comparison with calculated ones.
The $\mathrm{N}$-factor isocontours calculated for $x^{\prime} / c=0.483$ are presented in Fig. 5 along with the CF-mode frequencies selected for the main stability measurements. These preliminary calculations were made based on local linear stability theory (without surface curvature effects). The frequencies selected in the experiments are marked with vertical dashed-and-dotted lines. Red lines indicate positive frequencies, corresponding physically to the CF modes having positive spanwise wavenumbers and propagating opposite to the CF direction, while magenta lines indicate negative frequencies (in calculations), which correspond physically (in the experiment) to $\mathrm{CF}$ modes having negative spanwise wavenumbers and propagating in the $\mathrm{CF}$ direction. Zero-frequency modes can have various absolute values of the spanwise wavenumber. However, its sign is not defined uniquely because the stationary CF modes do not propagate in space. It is seen that there is a broad range of frequencies studied in the present instability experiments,

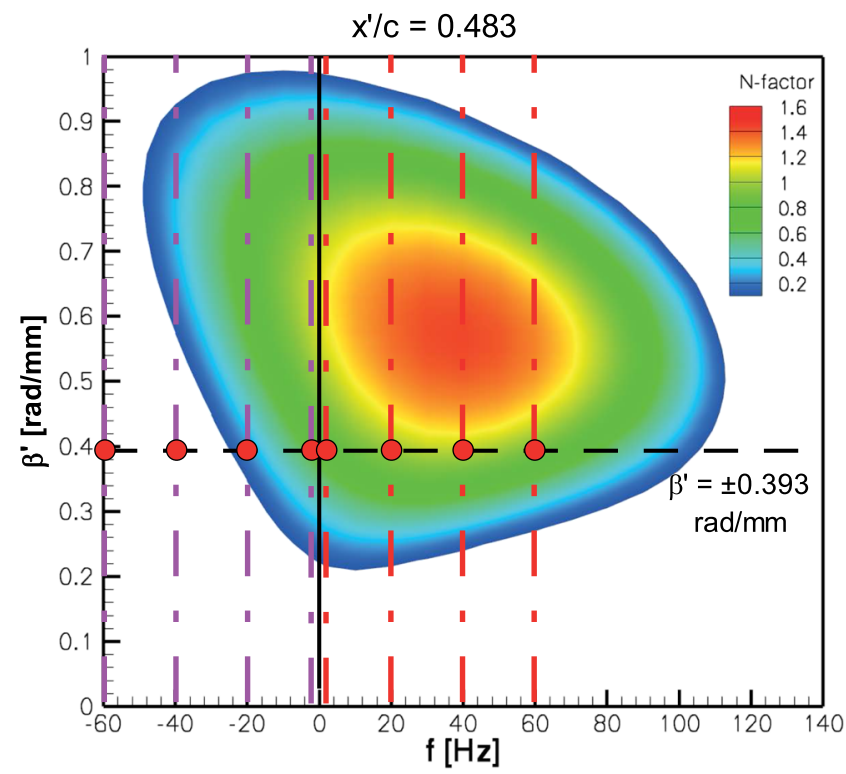

FIG. 5. Linear-stability diagram (disturbance amplitude $\mathrm{N}$-factors calculated at $x^{\prime} / \mathrm{c}$ $=0.483$ ) and disturbance frequencies selected for main CF-instability measurements. the majority of which correspond to amplified CF modes (according to the calculations).

The CF-instability problem has been studied experimentally for the following controlled parameters: (i) frequency of surface nonuniformities and excited CF waves, (ii) spanwise wavenumber (or scale) of surface nonuniformities and excited CF waves, and (iii) amplitude $\mathrm{E}$ of membrane excitation in arbitrary relative units controlled by a computer program used for signal generation. The complete list of the studied regimes is presented in Tables I and II.

The measured instability data were subjected to a deep mathematical processing. The instability characteristics were determined in Fourier space for every particular harmonic of the frequencyspanwise-wavenumber spectrum and, hence, they are independent of the specific way of excitation (as usual in the linear instability problem). The linearity of the problem under study was checked by collation of regime 3 against regime 3RS (here RS stands for Reduced Surface amplitude) indicated in Table II.

TABLE I. Studied CF-instability regimes with excitation of spanwise-periodic disturbances.

\begin{tabular}{lccc}
\hline \hline Names of regimes & $f_{\text {sur }}(\mathrm{Hz})$ & $\lambda_{z}^{\prime}(\mathrm{mm})$ & $\beta^{\prime}(\mathrm{rad} / \mathrm{mm})$ \\
\hline Regime M1 & 2 & 16 & \pm 0.393 \\
Regime M2 & 20 & 16 & \pm 0.393 \\
Regime M3 & 40 & 16 & \pm 0.393 \\
Regime M4 & 60 & 16 & \pm 0.393 \\
\hline \hline
\end{tabular}

TABLE II. Studied CF-instability regimes with excitation of spanwise-localized disturbances.

\begin{tabular}{lccc}
\hline \hline Names of regimes & $f_{\text {sur }}(\mathrm{Hz})$ & $E$ & $U / U_{e}$ \\
\hline Regime 1 & 2 (quasistationary case) & 10 & 0.79 \\
Regime 2 & 20 & 8 & 0.71 \\
Regime 3 & 40 & 5 & 0.68 \\
Regime 4 & 60 & 9 & 0.63 \\
Regime 3RS & 40 & 2.5 & 0.68 \\
\hline \hline
\end{tabular}




\section{BOUNDARY-LAYER DISTURBANCES AND THEIR STREAMWISE EVOLUTION}

\section{A. Physical nature of boundary-layer disturbances}

In order to investigate the physical nature of the excited boundary-layer disturbances and to select the best wall-normal distances for subsequent main instability measurements, a set of special tests has been performed in regimes with excitation of spanwiseperiodic disturbances (see Table I). The neighboring membranes oscillated in these regimes with opposite phases, hence leading to an excitation of a pair of boundary-layer disturbances with spanwise period $\lambda_{z}^{\prime}=16 \mathrm{~mm}$ and spanwise wavenumbers $\beta^{\prime}= \pm 0.393$ $\mathrm{rad} / \mathrm{mm}$.

Shown in Fig. 6 is a set of spanwise distributions of the boundary-layer disturbance amplitudes and phases measured at wall distance corresponding to $U / U_{e}=0.7$ (with constant wallnormal coordinate $y$ within every spanwise scan) at the downstream end of the region of stability measurements, i.e., at a distance $\Delta x=350 \mathrm{~mm}$ from the center of the disturbances source $\left(x^{\prime} / c=0.483\right)$. These measurements were performed in regimes M1, M2, M3, and M4. It is seen that these distributions are in a qualitative agreement with the stability diagram shown in Fig. 5. The corresponding excited frequency-wavenumber modes are marked in the stability diagram with red circles. Indeed, at high frequency (regime M4, $f=60 \mathrm{~Hz}$ ), the instability mode with negative spanwise wavenumber $\beta^{\prime}=-0.393 \mathrm{rad} / \mathrm{mm}$ (which corresponds to that with negative frequency on the theoretical diagram in Fig. 5) decays in agreement with theory. Consequently, at large streamwise coordinates (as in Fig. 6), only the oblique mode with positive spanwise wavenumber $\beta^{\prime}=+0.393 \mathrm{rad} / \mathrm{mm}$ survived, resulting in a constant amplitude and a linearly growing phase in spanwise direction (red lines and symbols).

Meanwhile, for the somewhat lower frequency of $40 \mathrm{~Hz}$ (regime M3) shown with brown lines and symbols in Fig. 8, a slight admixture of a weakly attenuating mode with spanwise wavenumber $\beta^{\prime}=-0.393 \mathrm{rad} / \mathrm{mm}$ is clearly observed, although its amplitude is small. At even lower frequency of $f=20 \mathrm{~Hz}$ (regime M2), the two modes with $\beta^{\prime}= \pm 0.393 \mathrm{rad} / \mathrm{mm}$ are within the instability regions (Fig. 5), although the mode $\beta^{\prime}=+0.393 \mathrm{rad} / \mathrm{mm}$ has significantly larger growth rates. In agreement with this, the corresponding distributions in Fig. 6 (light green color) become rather strongly modulated in the spanwise direction, displaying, however, average amplitude and phase growth characteristic for the predominant mode with $\beta^{\prime}=+0.393 \mathrm{rad} / \mathrm{mm}$.

Finally, at the lowest studied frequency of $2.0 \mathrm{~Hz}$ (regime M1) the two frequency-wavenumber modes become extremely close to each other at the stability diagram (Fig. 5). Accordingly, their amplification rates become very close to each other and the two modes have almost the same amplitudes at $x^{\prime} / c=0.483$, displaying a picture typical for a standing wave in the spanwise direction with characteristic deep amplitude modulation and $180^{\circ}$ phase jumps (dark green symbols and lines in Fig. 6).

As far as only one mode was present in the flow; in fact, at frequency of $60 \mathrm{~Hz}$ and $x^{\prime} / c=0.483$, the directly measured wall-normal profiles of the disturbance amplitude and phase corresponded exactly to the instability-mode eigenfunction, which is shown in Figs. 7(a) and 7(b) for amplitudes and phases of the streamwise velocity disturbances. At low frequencies, as at $f=2.0 \mathrm{~Hz}$, for instance, a simple procedure for the separation of the eigenfunctions of two excited oblique modes (with $\beta^{\prime}= \pm 0.393 \mathrm{rad} / \mathrm{mm}$ ) was performed as part of the experimental data processing. In this case, two wall-normal disturbance profiles were measured at two spanwise locations corresponding to the disturbance amplitude maximum and minimum, like those marked in Fig. 6 with vertical brown and blue dashed lines. Then, half-sum and half-difference of these profiles (in the plane of complex-valued amplitudes) provided the eigenfunctions of the corresponding pair of oblique normal modes. The eigenfunction obtained in this way for the mode with $\beta^{\prime}=+0.393$ $\mathrm{rad} / \mathrm{mm}$ at frequency $f=2.0 \mathrm{~Hz}$ is presented in Figs. 7(c) and 7(d) for amplitudes and phases of the streamwise velocity disturbances.

It is seen that the wall-normal profiles of the velocity disturbance amplitudes and phases obtained in this way are in a good agreement with eigenfunctions of the crossflow-instability modes calculated based on linear stability theories. The nonlocal stability theory provides better agreement with the measured profiles. The results of the present measurements performed for all studied frequencies have shown that the amplitude maxima of the CF modes with frequencies $f=2,20,40$, and $60 \mathrm{~Hz}$ (regimes M1, M2, M3,

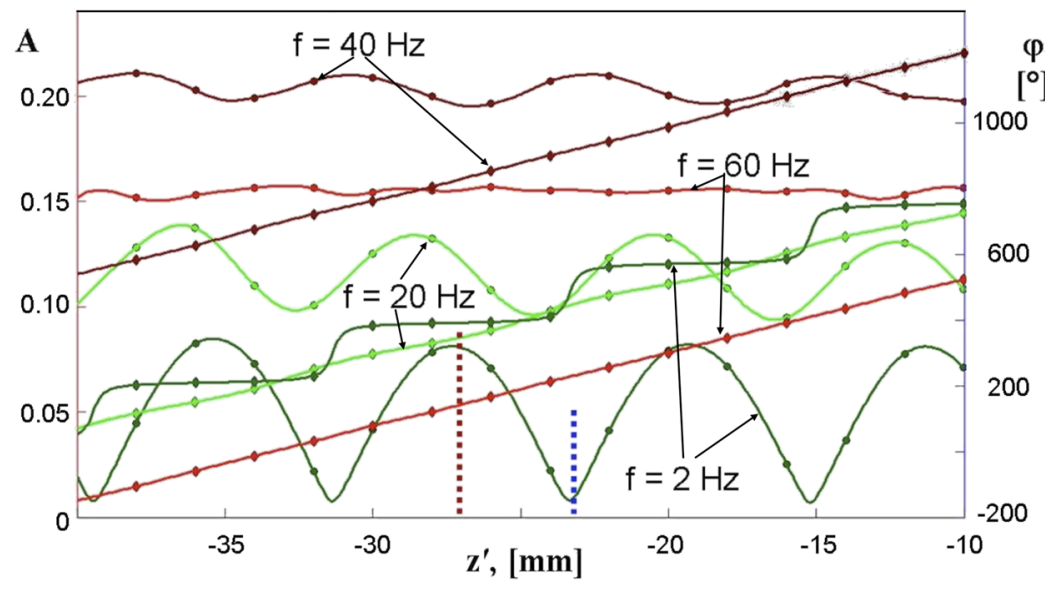

FIG. 6. Spanwise distributions of boundary-layer disturbance amplitudes and phases measured in regimes M1, $M 2, M 3$, and M4 at the end of the region of stability measurements $\left(x^{\prime} / c=0.483\right)$ at $U / U_{e}=0.7$. 

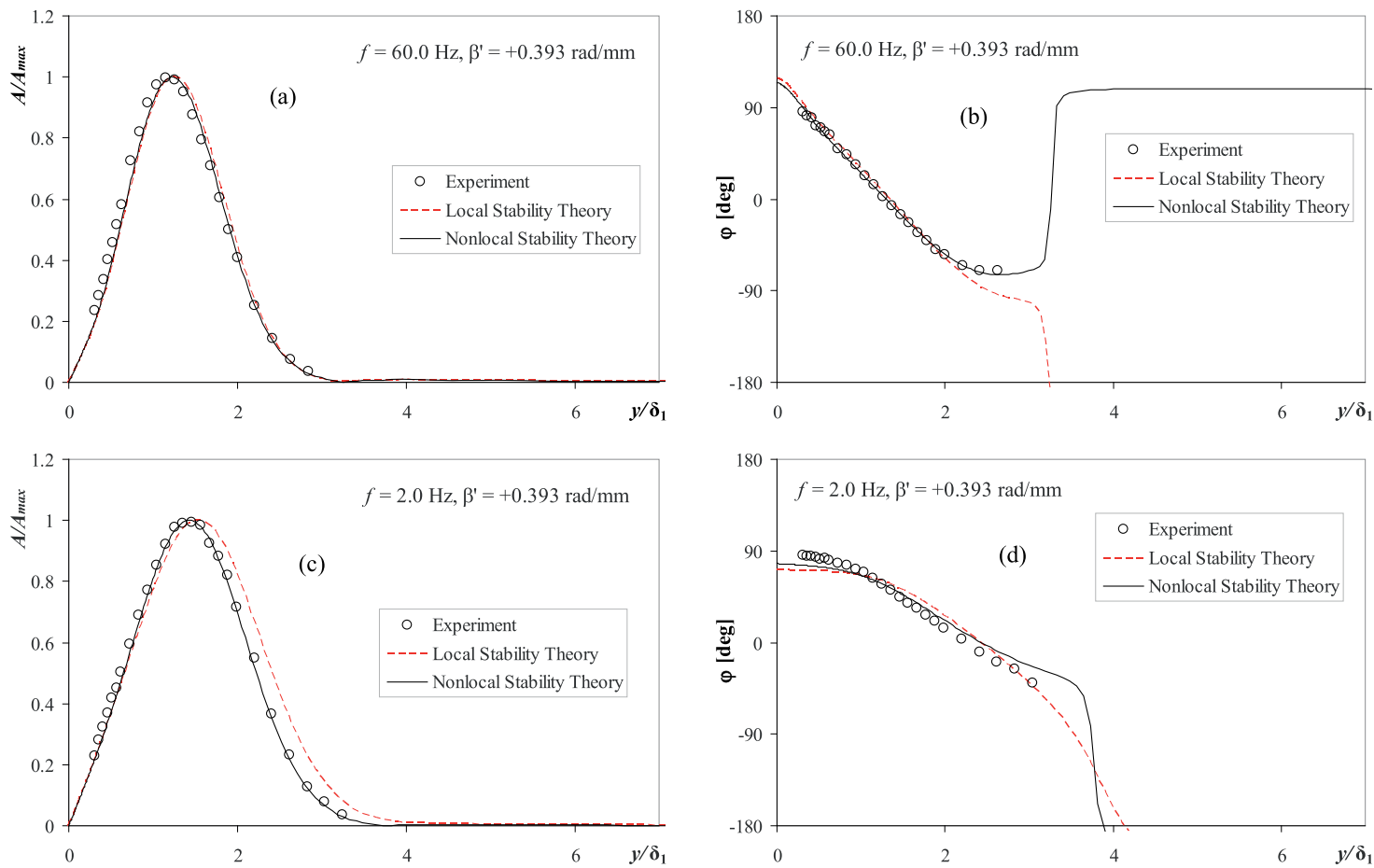

FIG. 7. Wall-normal amplitude [(a) and (c)] and phase [(b) and (d)] profiles of normal oblique instability modes (eigenfunctions) measured in regime $\mathrm{M} 4$ at frequency $f=60 \mathrm{~Hz}$ $[(a)$ and $(b)]$ and in regime $\mathrm{M} 1$ at frequency $f=2.0 \mathrm{~Hz}[(\mathrm{c})$ and $(\mathrm{d})]$ for spanwise wavenumber $\beta^{\prime}=+0.393 \mathrm{rad} / \mathrm{mm}$ at $x^{\prime} / c=0.483$ in comparison with CF-mode eigenfunctions calculated based on locally-parallel (LST) and nonlocal (PSE) stability theory.

and M4, respectively) are observed at wall-normal distances, which correspond to the values of $U / U_{e}$ of $0.79,0.71,0.68$, and 0.63 , respectively. This result is in a good agreement with several previous observations related to the CF-mode eigenfunctions (see, e.g., Refs. 42, 63, and 64). It is also known that the shapes of the CF-mode amplitude profiles depend on the spanwise wavenumber and the streamwise coordinate rather weakly. Therefore, we have used the values of the dimensionless wall-normal distances $U / U_{e}$ indicated above in all our main stability measurements described below.

Based on the results described above, a conclusion was made that the boundary-layer perturbations excited by the used disturbances source are the unsteady CF-instability modes.

\section{B. Linearity of CF-instability problem under study}

The independence of the measured CF-instability characteristics from the disturbance amplitude was investigated in a pair of regimes 3 and 3RS (see Table II). All parameters of the base flow and the excited disturbances were identical in these two regimes except for the amplitudes of excitation $E$ of the disturbances. One level of membrane excitation differed from another one by a factor of two ( $E$ was equal to either 5.0 or 2.5). Two pairs of spanwise distributions of disturbance amplitudes and phases were measured inside the boundary layer at two chordwise coordinates: $x^{\prime} / c=0.198$ and 0.483 (i.e., at $x_{s}^{\prime}=170.8$ and $399.6 \mathrm{~mm}$ ); the first of which corresponds to an initial chordwise location, while the second one to the end of the region of main stability measurements. These distributions are presented in Fig. 8 together with the same distributions obtained at $x^{\prime} / c=0.483$ but normalized by the spanwiseaveraged values of disturbance amplitudes and phases measured at the initial section $x^{\prime} / c=0.198$. The averaging was performed only for those points in which the accuracy of measurements was reliable due to large enough disturbance amplitudes. The directly measured amplitude distributions were divided by the corresponding averaged amplitude, while the average phases were subtracted from the directly measured phase distributions. Namely, the resulting normalized amplitudes and phases were found as $A_{n}\left(z^{\prime}\right)=A\left(x^{\prime} / c\right.$ $\left.=0.483, z^{\prime}\right) / \operatorname{mean}\left\{A\left(x^{\prime} / c=0.198, z^{\prime}\right)\right\}$ and $\varphi_{n}\left(z^{\prime}\right)=\varphi\left(x^{\prime} / c=0.483, z^{\prime}\right)$ $-\operatorname{mean}\left\{\varphi\left(x^{\prime} / c=0.198, z^{\prime}\right)\right\}$. Hence, the normalized amplitude and phase distributions shown in Fig. 8 reflect purely the characteristics of development of the boundary-layer disturbances, i.e., the instability characteristics of the flow measured in regimes 3 and 3RS. The coincidence of the normalized distributions obtained at two different levels of excitation indicates that the measured instability characteristics are independent of the excitation amplitudes used, i.e., that the measurements were indeed performed in the linear regime of disturbance development.

\section{Evolution of CF-wave trains}

Two examples of sets of spanwise distributions of the disturbance amplitudes and phases within wave trains of CF-instability modes measured in regimes 4 and 1 at frequencies $f=60$ and $2.0 \mathrm{~Hz}$, respectively, are presented in Figs. 9(a) and 9(b), and Figs. 10(a) and 

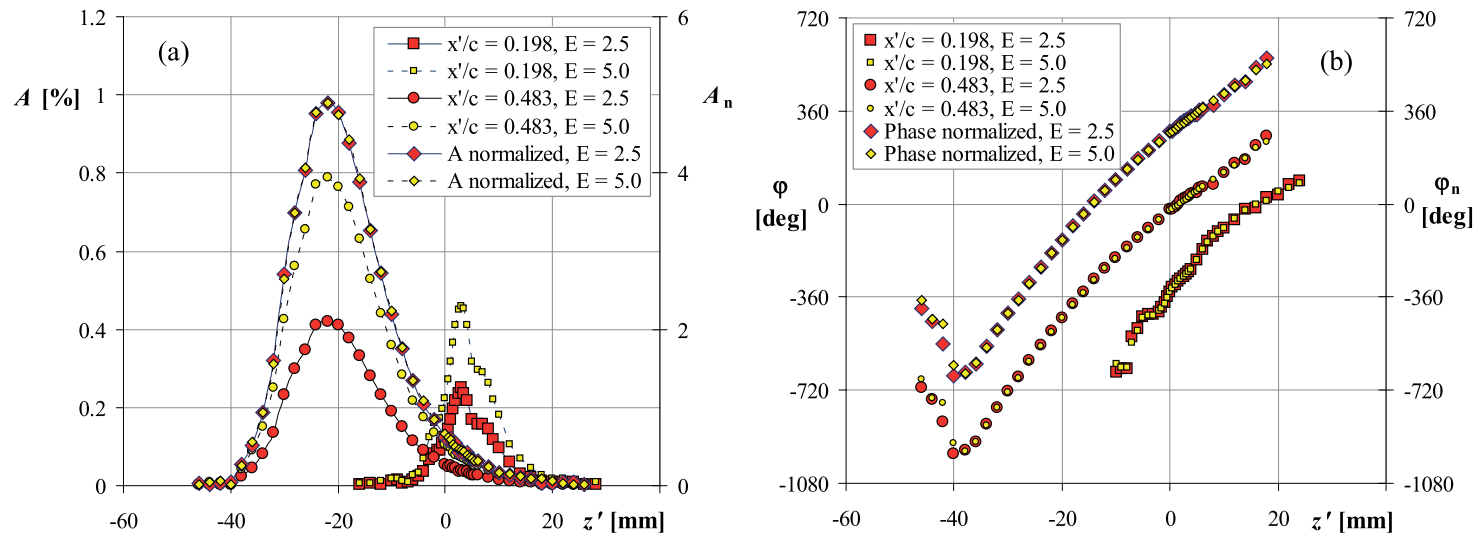

FIG. 8. Spanwise distributions of disturbance amplitudes (a) and phases (b) measured directly in regimes 3 and $3 R S$ for two different amplitudes of disturbance excitation $\mathrm{E}=5$ and 2.5 at two chordwise locations $x^{\prime} / c=0.198$ and 0.483 . $f=40 \mathrm{~Hz}$ and the corresponding distributions obtained at $x^{\prime} / c=0.483$ normalized by mean values measured at initial section $x^{\prime} / c=0.198$.

10(b). The measurements are performed for the spanwise-localized excitation (one membrane is activated) with a broad spectrum of spanwise wavenumbers $\beta^{\prime}$. It is seen that the shape of the distributions is significantly different for these two frequencies. It displays a rather smooth $z^{\prime}$-behavior for high frequency [Figs. 9(a) and 9(b)] and a picture with rapid variation of amplitudes and phase jumps at low, quasistationary frequency [Figs. 10(a) and 10(b)].

The corresponding instantaneous shapes of the wave trains, reconstructed based on the experimental data, are shown in Figs. 9(c) and 10(c) (Multimedia view) for the same two frequencies. (These figures are linked to the attached files displaying an animated behavior of the waves.) It is seen, in particular, that the traveling CF waves at frequency $f=60 \mathrm{~Hz}$ [Fig. 9(c) (Multimedia view), regime 4] propagate in one direction at angle of about $80^{\circ}$ to the $x$-axis and at angle of about $115^{\circ}$ to the $x^{\prime}$-coordinate, i.e., toward the airfoil leading edge. Meanwhile, at very low, quasistationary frequency $f=2.0 \mathrm{~Hz}$ [Fig. 10(c) (Multimedia view), regime 1], the animated disturbance displays clearly the standing-wavelike oscillation because it is composed of two waves propagating in the opposite directions along the $z^{\prime}$-axis.

\section{Spanwise-wavenumber spectra and evolution of normal CF modes}

The distributions like those shown in Figs. 9(a), 9(b), 10(a), and 10(b) have been subjected to a spanwise Fourier decomposition for obtaining the spanwise-wavenumber disturbance spectra in regimes $1,2,3$, and 4 with spanwise-localized disturbance excitation. The amplitude parts of these spectra are illustrated in Figs. 11(a), 11(c), 11(e), and 11(g). The corresponding streamwise distributions of normal CF-mode amplitudes and phases obtained from the spanwise-wavenumber spectra are illustrated in Figs. 11(b), 11(d), 11(f), and 11(h) for several values of the spanwise wavenumber and are compared with the corresponding calculated distributions. Note that the phase distributions $\tilde{\phi}$ shown in Figs. 11(b), 11(d), $11(\mathrm{f})$, and $11(\mathrm{~h})$ are defined in a nonorthogonal $\left(x, z^{\prime}\right)$ coordinate system, in which the $z^{\prime}$-axis origin is located on the $x$-coordinate. The use of this reference frame is convenient during measurements because the directions of the group velocity vectors are much closer to the direction of the $x$-axis rather than of the $x^{\prime}$-axis and, hence, the phases $\tilde{\phi}$ have the $2 k \pi$ uncertainties, occurred at the streamwise displacement, with much lower integer values of $k$ compared to the Cartesian $\left(x^{\prime}, z^{\prime}\right)$-coordinate system. In total, such distributions were obtained for every particular fixed value of the spanwise wavenumber $\beta^{\prime}$ and frequency $f$. It is important to note that there is a very good agreement between all shown experimental and theoretical chordwise distributions.

The spectra shown in Figs. 11(a), 11(c), 11(e), and 11(g) display the spanwise-wavenumber ranges of CF modes developing in the flow. In particular, at high frequency [Fig. 11(a), regime 4], only modes with positive spanwise wavenumbers exist. These modes propagate against the cross-flow direction. Meanwhile, at lower frequencies [Figs. 11(b), 11(d), and 11(f), regimes 3, 2, and 1, respectively], the modes with both positive and negative spanwise wavenumbers are present in the flow. The lower the frequency is, the larger are the relative amplitudes of the CF modes having negative values of $\beta^{\prime}$. These results are also in a good qualitative agreement with the stability diagram shown in Fig. 5 , as well as with the discussion above related to Fig. 6 obtained for spanwise periodical excitation.

For the highest frequency studied $(f=60 \mathrm{~Hz})$, two examples of the CF-mode amplification curves are presented in Fig. 11(b) for spanwise wavenumbers $\beta^{\prime}=+0.209$ and $+0.419 \mathrm{rad} / \mathrm{mm}$. These spanwise wavenumbers correspond to either decaying $\left(\beta^{\prime}=+0.209\right.$ $\mathrm{rad} / \mathrm{mm})$ or amplifying $\left(\beta^{\prime}=+0.419 \mathrm{rad} / \mathrm{mm}\right)$ perturbations.

At a frequency of $40 \mathrm{~Hz}$ the growth rates of the most amplified CF modes with positive spanwise wavenumber [Fig. 11(d)] remains close to those observed at $60 \mathrm{~Hz}$ [Fig. 11(b)]. However, in contrast to the highest frequency case, some CF waves having negative spanwise wavenumbers become measurable although they display a nearly neutral behavior initially and attenuate further downstream [Fig. 11(d)]. Meanwhile, at even lower frequency (of $20 \mathrm{~Hz}$ ), amplitudes of the CF modes with negative values of $\beta^{\prime}$ become much larger [Fig. 11(e)] and are able even to display weak amplification [Fig. 11(f)] although their growth rates are very weak. 

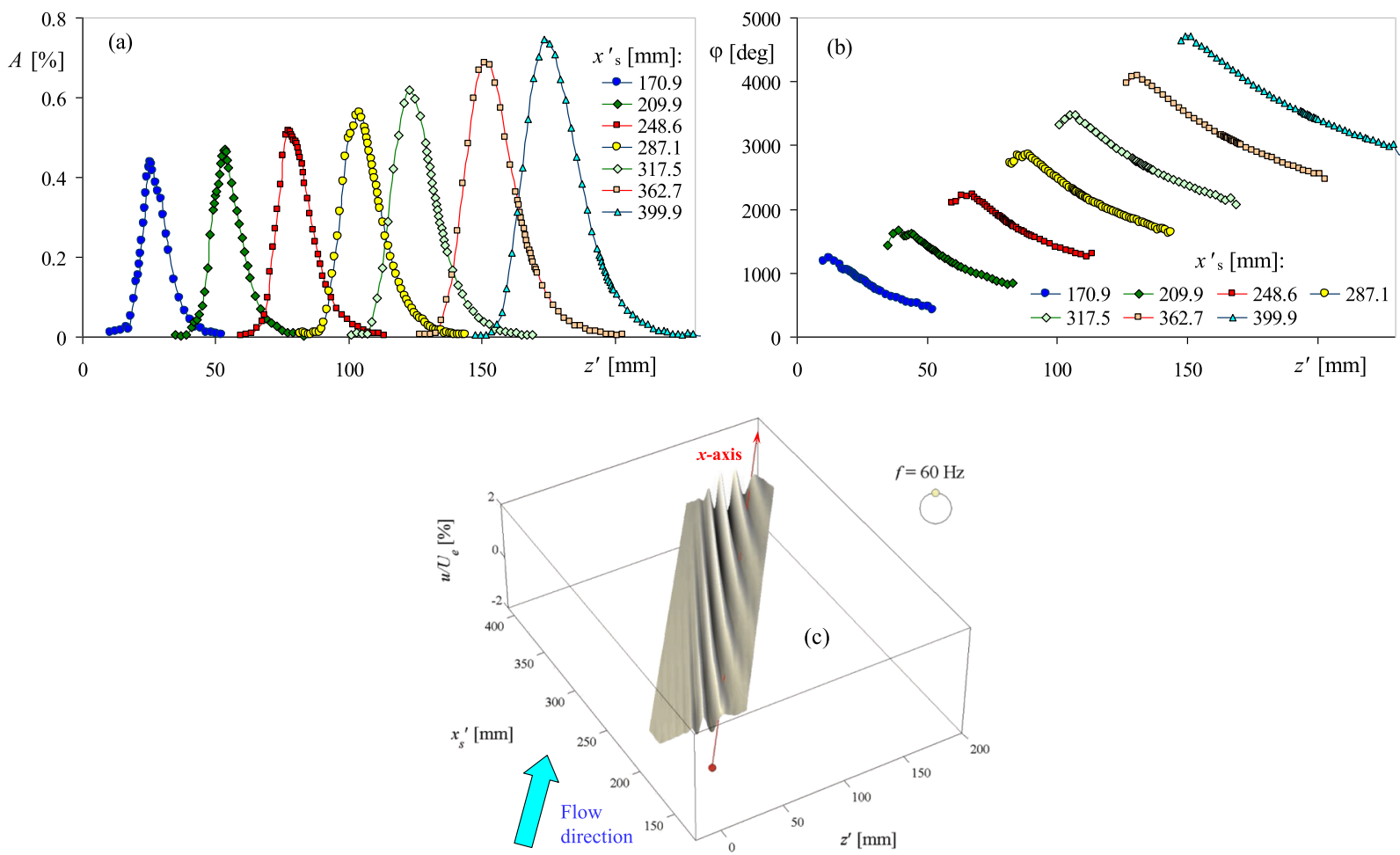

FIG. 9. Set of spanwise distributions of disturbance amplitudes (a) and phases (b) within wave train (c) of CF-instability modes measured in regime 4 at frequency $f=60 \mathrm{~Hz}$ for spanwise-localized excitation with a broad spectrum of spanwise wavenumbers $\beta^{\prime}$. Multimedia view: https://doi.org/10.1063/1.5094609.1

At the lowest frequency studied $(f=2.0 \mathrm{~Hz})$, the CFwave amplitude development, measured and calculated for the most quickly growing modes with positive and negative spanwise wavenumbers $\left(\beta^{\prime}= \pm 0.419 \mathrm{rad} / \mathrm{mm}\right)$, is very similar for both modes [Fig. 11(h)]. The spanwise-wavenumber spectra become quasisymmetric [Fig. 11(g)]. However, the corresponding phase distributions [Fig. 11(h)] show that these modes propagate at different angles relative to the flow direction. Note that for a frequency tending to zero, these two modes become indistinguishable from each other and correspond to a stationary CF mode with undefined propagation direction and sign of the spanwise wavenumber. The low-frequency regime with $f=2.0 \mathrm{~Hz}$ corresponds to a quasistationary one because during one period of the CF-mode oscillation the flow passes the whole region of measurements more than 30 times.

More detailed comparisons of the amplification curves measured and calculated for great number of values of the spanwise wavenumber for all studied frequencies have shown a very good agreement for the whole broad frequency-wavenumber spectrum studied. According to our knowledge, such good agreement had never been achieved previously for unsteady CF modes in sweptwing boundary layers on a real airfoil. This agreement substantiates the applicability of the linear stability theory for the description of the initial stages of the laminar-turbulent transition occurring in a cross-flow dominated boundary layer on a real airfoil section.

\section{E. Measured phase speeds and increments of normal CF modes}

Differentiation of measured streamwise phase distributions provided (after some transforms of the coordinate systems) the dispersion curves $\alpha_{r}^{*}\left(\beta^{*}\right)$ for every particular frequency $f$, as well as the corresponding CF-wave propagation angles $\theta^{*}\left(\beta^{*}\right)$. Such distributions, obtained in a range of streamwise coordinates $x_{s}^{\prime}$ from 171 to $400 \mathrm{~mm}$, are illustrated in Figs. 12(a) and 12(b) and Figs. 12(c) and $12(\mathrm{~d})$, for regimes 4 and 1 with frequencies of 60 and $2.0 \mathrm{~Hz}$, respectively. It is seen that essentially unsteady modes (regime 4 , $f=60 \mathrm{~Hz}$ ) propagate in a broad range of wave angles $\theta^{*}$ between approximately $\pm 90^{\circ}$ [Fig. 12(b)] although modes with negative spanwise wavenumbers have extremely low amplitudes [Fig. 11(a)]. The streamwise wavenumbers $\alpha_{r}^{*}$ decrease with chordwise coordinate for positive spanwise wavenumbers and increase for negative ones [Fig. 12(a)]. In the whole presented range of values of $\beta^{*}$ (between $\pm 1 \mathrm{rad} / \mathrm{mm}$ ), the streamwise wavenumbers are always positive.

At a somewhat lower frequency of $40 \mathrm{~Hz}$ (regime 3), the streamwise wavenumbers $\alpha_{r}^{*}$ are reduced and even become equal to zero or negative at $x_{s}^{\prime}=171 \mathrm{~mm}$ for large negative values of the spanwise wavenumber $\beta^{*}$. These waves propagate upstream the local potential flow taken at the boundary-layer edge. At even lower frequency of $20 \mathrm{~Hz}$, almost all CF-instability modes with negative values of $\beta^{*}$ have negative values of $\alpha_{r}^{*}$, while at $f=2.0 \mathrm{~Hz}$, this property is 

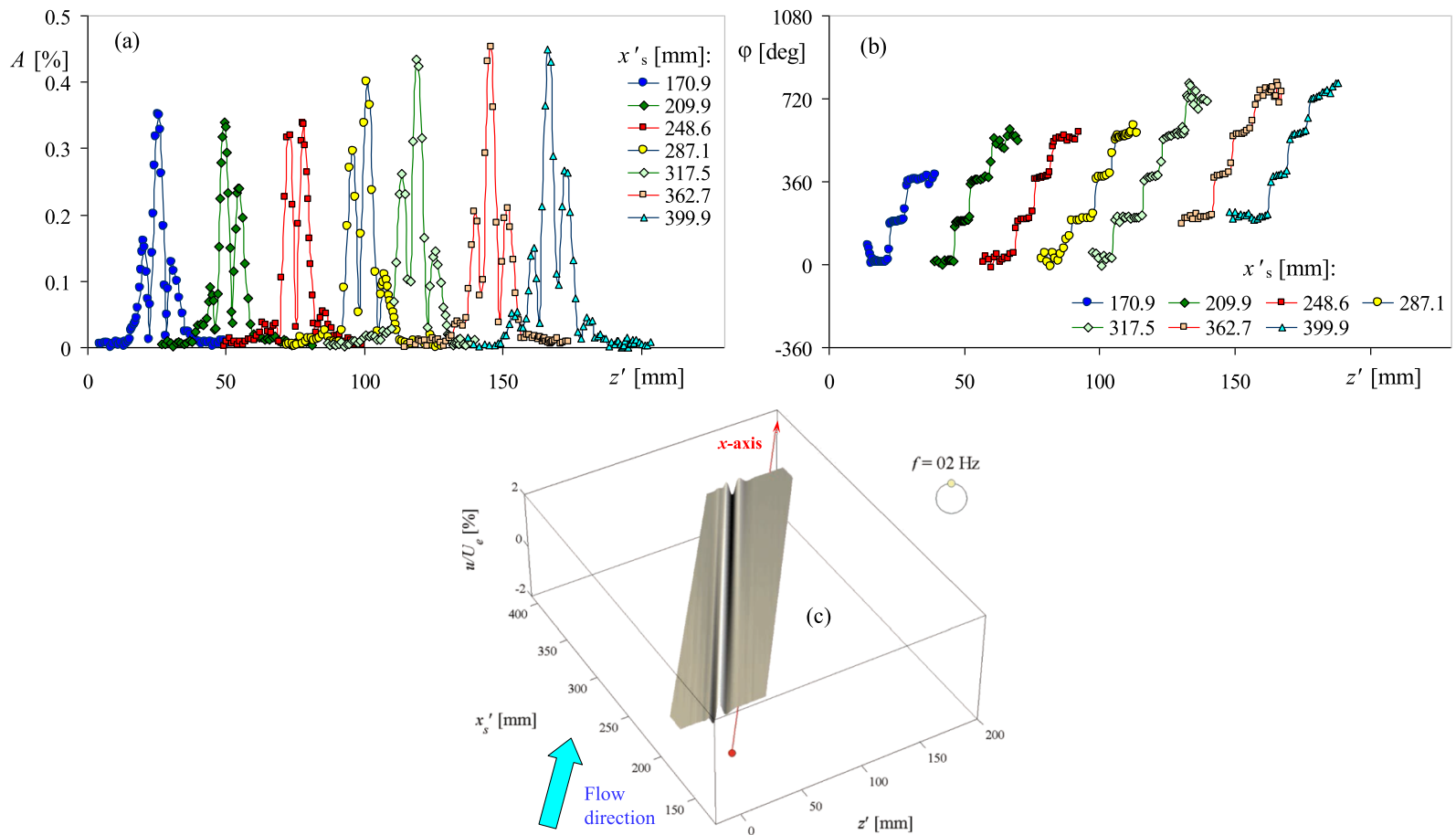

FIG. 10. Set of spanwise distributions of disturbance amplitudes (a) and phases (b) within wave train (c) of CF-instability modes measured in regime 1 at quasistationary frequency $f=2.0 \mathrm{~Hz}$ for spanwise-localized excitation with a broad spectrum of spanwise wavenumbers $\beta^{\prime}$. Multimedia view: https://doi.org/10.1063/1.5094609.2

inherent for all modes with $\beta^{*}<0$ and all values of the streamwise coordinate $x_{s}^{\prime}$ [Fig. 12(c)].

At a very low frequency of $2.0 \mathrm{~Hz}$, the disturbances represent almost stationary (quasistationary) CF vortices with wavevectors inclined at angles of either about +84 to $+87^{\circ}$ (at $\beta^{*}>0$ ) or about -92 to $-97^{\circ}\left(\right.$ at $\left.\beta^{*}<0\right)$ relative to the local potential-flow direction [Fig. 12(d)]. (Note that a mode with $\beta^{*}=0 \mathrm{rad} / \mathrm{mm}$ does not exist at zero frequency.) These angles correspond approximately to the orientation of the CF-vortex axes (or to wave-fronts of the quasisteady modes) of about $2-3^{\circ}$ relative to the $x^{*}$-axis, which is typical for stationary CF modes. Meanwhile, at high frequencies, the CF modes are able to propagate at almost any inclination angle [as seen in Fig. 12(b)].

Two kinds of phase speeds of the CF-instability modes have been obtained experimentally: (i) the phase speed $C_{K}=2 \pi f /|\mathbf{K}|$ in the direction of wavevectors $\mathbf{K}$ and (ii) the phase speed $C_{x^{*}}=2 \pi f / \alpha_{r}^{*}$ in the direction of the local potential flow velocity vector (i.e., in the $x^{*}$-direction). These two speeds are illustrated in Figs. 13(a) and 13(b), respectively, vs the spanwise wavenumber $\beta^{*}$ for all studied frequencies (regimes 4, 3, 2, and 1) at one of the streamwise locations (with $x_{s}^{\prime}=287.1 \mathrm{~mm}$ ).

It is seen in Fig. 13(a) that at high frequencies and spanwise wavenumbers close to zero, the wave fronts propagate rather quickly (with a speed of about $0.30-0.4$ of $U_{e}$ ). However, the propagation velocity $C_{K}$ drops down rapidly when $\left|\beta^{*}\right|$ increases. These velocities are also significantly reduced when the disturbance frequency tends to zero. In the quasistationary regime 1 (with $f=2.0 \mathrm{~Hz}$ ), an averaged value of $C_{K}$ is as low as of about 0.002 of $U_{e}$, i.e., the waves almost stop.

The phase speeds in the $x^{*}$-direction show a rather different behavior [Fig. 13(b)] depending on frequency. They have asymptotic discontinuities at certain negative values of the spanwise wavenumber $\beta^{*}$, whose absolute value increases with frequency. When approaching such discontinuity at reduction of $\beta^{*}$, the phase speed $C_{x^{*}}$ tends from moderate positive values to $+\infty$ and comes back from $-\infty$ approaching some moderate negative values. It is important to note that such behavior is physically reasonable and does not contradict to physical sense. It means that the wave front orientation changes with the spanwise wavenumber and becomes parallel to the $x^{*}$-axis in the asymptotic discontinuity point.

Differentiation of the experimental CF mode amplification curves [like those shown in Figs. 11(b), 11(d), 11(f), and 11(h)] provides the spatial growth rates $-\alpha_{i}^{\prime}$ of the normal CF modes in the chordwise direction, which are shown in Figs. 14(a) and 14(b), Figs. 14(c) and 14(d), and Figs. 14(e) and 14(f) for frequencies $f=60,40$, and $2.0 \mathrm{~Hz}$, respectively, vs the spanwise wavenumber $\beta^{*}$ [Figs. 14(a), 14(c), and 14(e)] and vs wave propagation angle $\theta^{*}$ [Figs. 14(b), 14(d), and 14(f)]. The growth rates were obtained as derivatives of quadratic or linear approximation of experimental amplification curves in the logarithmic scale. The linear approximation was used only in cases of large scattering of experimental points, which led to an inapplicability of the parabolic approximation. In these cases, streamwise averaged only were estimated. 

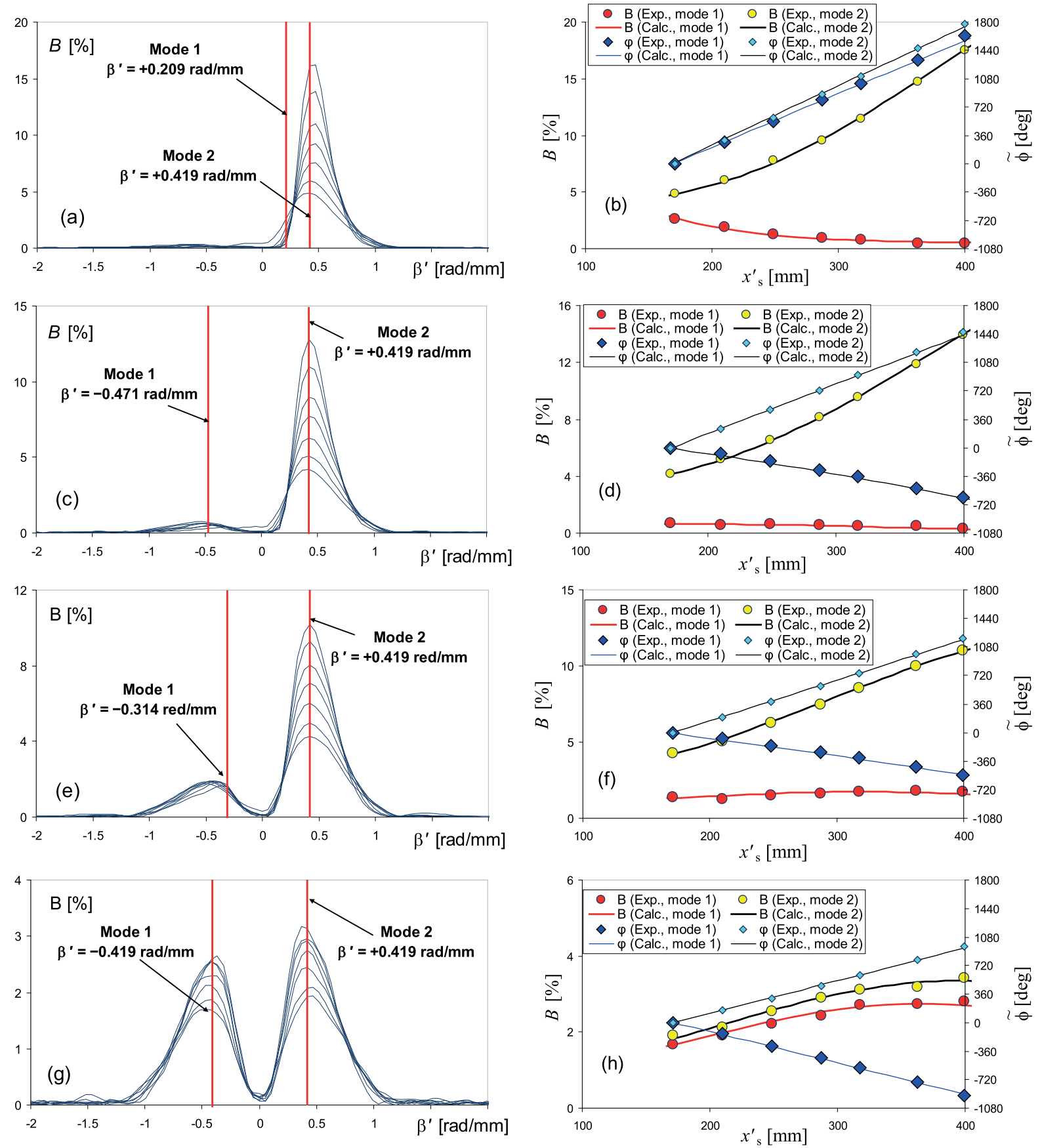

FIG. 11. Spanwise-wavenumber spectra of instability modes measured at various chordwise positions for $f=60,40,20$ (a) and (d) $2.0 \mathrm{~Hz}$ [(a), (c), (e), (g), respectively] and chordwise distributions of spectral amplitudes and phases for the same frequencies [(b), (d), (f), and (h)] measured and calculated for several spanwise wavenumbers marked with vertical lines in plots (a), (c), (e), and (g).

In agreement with the previously discussed experimental and theoretical results, only waves with positive spanwise wavenumbers, i.e., those propagated upstream the cross flow, are amplified at the highest studied frequency of $60 \mathrm{~Hz}$ [Figs. 11(a) and 11(b)].
Due to the very rapid attenuation of modes with negative values of $\beta^{*}$ at this high frequency, it was impossible to obtain experimentally the corresponding growth rates, which are thus absent in Fig. 14(a). At somewhat lower frequency $(f=40 \mathrm{~Hz})$, a very 

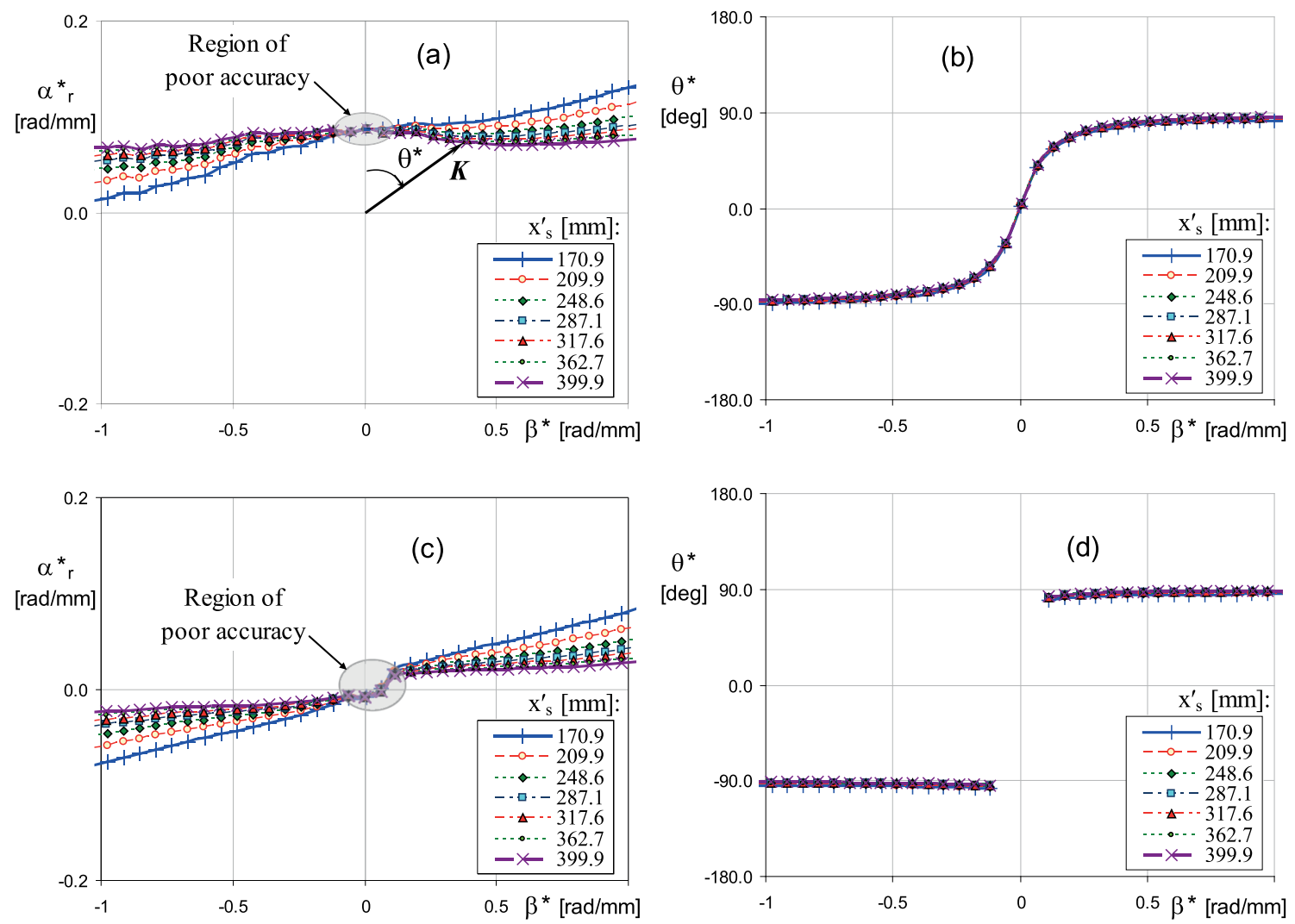

FIG. 12. Streamwise wavenumber $\alpha_{r}^{*}\left[(\mathrm{a})\right.$ and (c)] and wave propagation angle $\theta^{*}\left[(\mathrm{~b})\right.$ and (d)] of normal CF modes vs spanwise wavenumber $\beta^{*}$ measured in local coordinate system at various chordwise coordinates $x_{s}^{\prime}$ for frequencies $f=60[(\mathrm{a})$ and (b)] and $f=2.0 \mathrm{~Hz}[(\mathrm{c})$ and (d)].

small range of weakly amplified CF waves appears at negative values of $\beta^{*}$ [Fig. 14(c)]. At a frequency of $20 \mathrm{~Hz}$, this range becomes much broader, and at the lowest studied frequency $f=2.0 \mathrm{~Hz}$ (the quasistationary case), the spanwise-wavenumber distributions of the amplification rates become almost symmetric with respect to the point $\beta^{*}=0$ [Fig. 14(e)]. Note that for exactly stationary modes (i.e., for $f=0$ ), these distributions must be fully symmetric ones because there is no difference for them between negative and
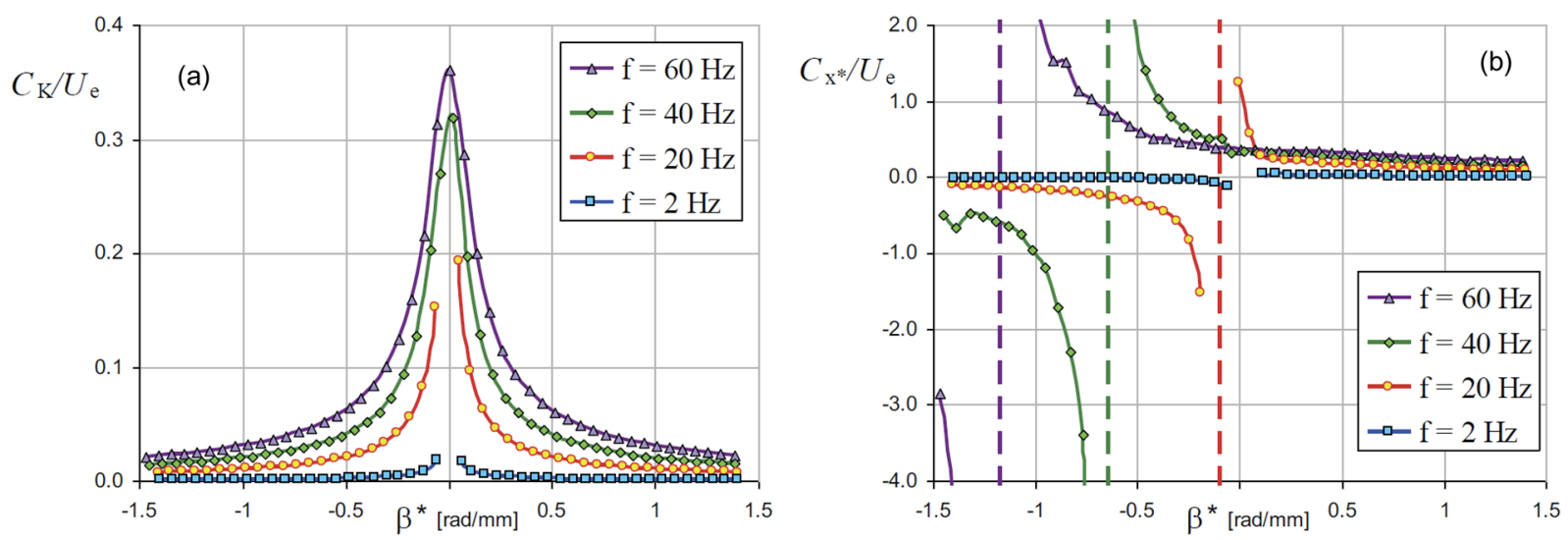

FIG. 13. Phase velocities $C_{K}$ in the direction of wavevectors (a) and $C_{x^{*}}$ in $x^{*}$-direction (b) vs spanwise wavenumber $\beta^{*}$ for normal CF modes with frequencies of regimes $4,3,2$, and 1 measured at $x_{s}^{\prime}=287.1 \mathrm{~mm}$. Vertical dashed lines show approximate locations of asymptotic discontinuities. 

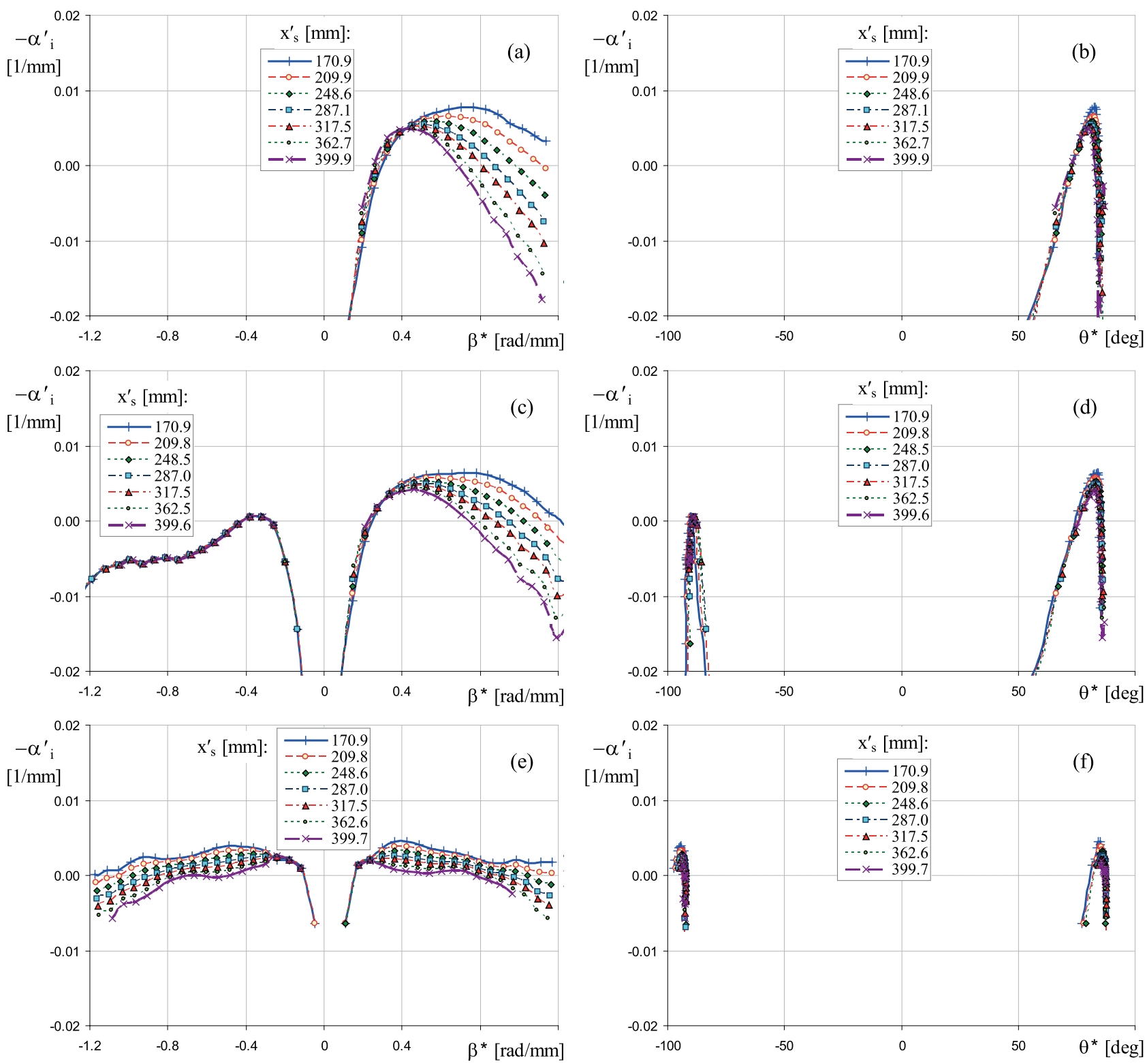

FIG. 14. Spatial growth rates of normal CF modes vs spanwise wavenumber [(a), (c), and (e)] and wave propagation angle $[(b)$, (d), and (f)] measured at various chordwise coordinates $x_{s}^{\prime}$ for frequencies: $f=60[(\mathrm{a})$ and $(\mathrm{b})], f=40[(\mathrm{c})$ and $(\mathrm{d})]$, and $f=2.0 \mathrm{~Hz}[(\mathrm{e})$ and $(\mathrm{f})]$.

positive spanwise wavenumbers as far as these modes do not propagate in space but stand still.

It is also seen in Figs. 14(a), 14(c), and 14(e) that for positive spanwise wavenumbers, the largest growth rates of the CFmodes are observed for the highest studied frequencies $(f=60$ and $40 \mathrm{~Hz}$ ) and the amplification gets weaker when the frequency is reduced. This is a rather typical property of the CF-instability modes: the most amplified modes are always nonstationary ones.
Figures 14(b), 14(d), and 14(f) display the dependence of the growth rates on the wave propagation angle $\theta^{*}$. At the highest studied frequency, the amplified CF waves propagate at angles between $73^{\circ}$ and $83^{\circ}$ relative to the boundary-layer edge streamline [Fig. 14(b)]. With reduction of the frequency [Figs. 14(d) and 14(f)], this range becomes narrower (for the most dangerous CFmodes, which have positive spanwise wavenumbers and propagated upstream the cross flow). For quasisteady modes of frequency $2.0 \mathrm{~Hz}$, this range of angles concentrates in a very narrow domain 

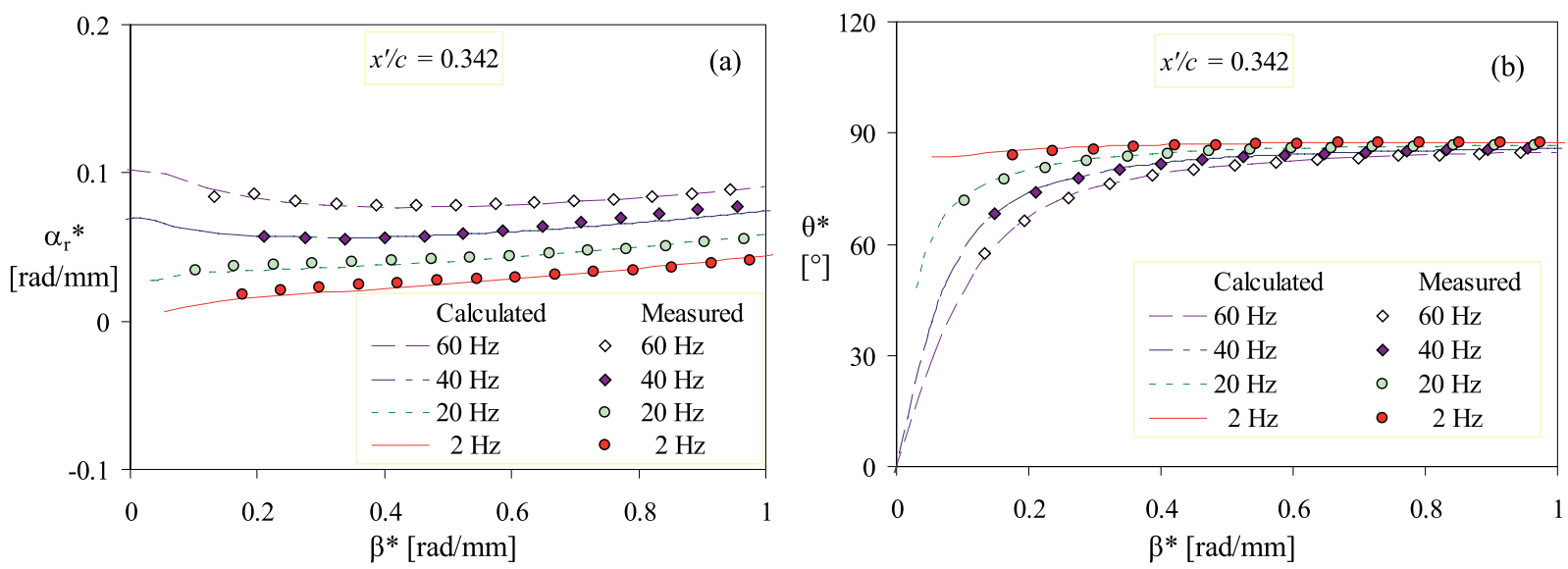

FIG. 15. Comparison of measured and calculated values of streamwise wavenumber $\alpha_{r}^{*}$ (a) and wave propagation angle $\theta^{*}$ (b) of normal CF modes vs spanwise wavenumber $\beta^{*}$ obtained for all studied frequencies at chordwise coordinate $x_{s}^{\prime}=287.1 \mathrm{~mm}$.

between $84^{\circ}$ and $87^{\circ}$ [Fig. 14(f)]. For negative spanwise wavenumbers (i.e., for the CF modes propagated in the cross-flow direction) the range of the amplified CF waves is always rather narrow and is located near values of $\theta^{*}$ close to $-90^{\circ}$ (from about $-88^{\circ}$ for $f=40 \mathrm{~Hz}$ to about $-94^{\circ}$ for $f=2.0 \mathrm{~Hz}$ ).

\section{F. Comparison of measured and calculated dispersion characteristics of normal CF modes}

Comparisons of distributions of the streamwise wavenumbers $\alpha_{r}^{*}$ and the wave propagation angles $\theta^{*}$ vs the spanwise wavenumber $\beta^{*}$ measured and calculated for all four frequencies in the middle of the studied range of the streamwise coordinate at $x_{s}^{\prime}=287.1 \mathrm{~mm}$ $\left(x^{\prime} / c=0.342\right)$ are illustrated in Figs. 15(a) and 15(b), respectively. Comparisons of the corresponding distributions of the phase velocities $C_{K}$ and $C_{x^{*}}$ are presented in Figs. 16(a) and 16(b). These results are shown for positive values of the spanwise wavenumber $\beta^{*}$, which include the most amplified CF-instability modes.

A very good agreement between the measured and calculated dispersion curves is observed for all studied frequencies and spanwise wavenumbers [Fig. 15(a)]. It is seen that the streamwise wavenumber of the unsteady CF-instability modes increases with frequency and depends rather weakly on the spanwise wavenumber. Meanwhile, the CF-wave propagation angle decreases with frequency for all values of $\beta^{*}$ [Fig. 15(b)]. The quasisteady CF modes (with frequency of $2 \mathrm{~Hz}$ ) have propagation angles close to $87.5^{\circ}$. This angle only slightly diminishes with the reduction of the spanwise wavenumber.

The two CF-wave phase speeds defined above, determined both in the wavevector direction [speed $C_{K}$, Fig. 16(a)] and in the $x^{*}$ direction [speed $C_{x^{*}}$, Fig. 16(b)] also demonstrate a very good agreement between the experimental and theoretical results for all
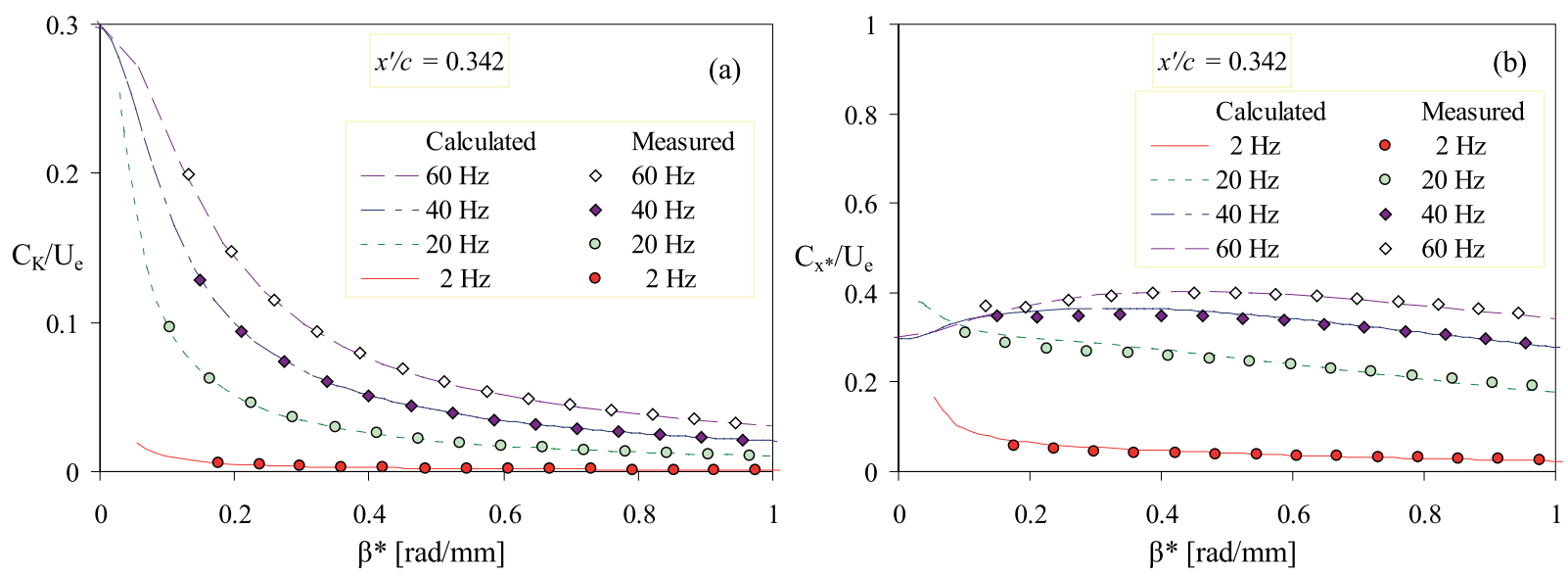

FIG. 16. Comparison of measured and calculated dimensionless phase velocities $C_{K}$ in the direction of wavevectors (a) and $C_{x^{*}}$ in the $x^{*}$ direction (b) presented for normal CF modes vs spanwise wavenumber $\beta^{*}$ obtained for all studied frequencies at chordwise coordinate $x_{s}^{\prime}=287.1 \mathrm{~mm}$. 
studied frequencies and spanwise wavenumbers. The two phase speeds increase with frequency. The speeds of the quasisteady CF mode (with $f=2 \mathrm{~Hz}$ ) become very low and is approaching to the zero value characteristic for stationary CF vortices. In other words, the traveling $\mathrm{CF}$ waves decelerate with reduction of frequency and come to a stop when $f \rightarrow 0$.

The very good agreement obtained for all dispersion characteristics of the measured and calculated CF-instability modes clearly demonstrates the applicability of nonlocal stability theory (PSE) and its high accuracy in analyzing the linear instability characteristics of the swept-wing boundary layer under study and indicates that the boundary-layer disturbances measured experimentally do correspond to this particular kind of the boundary-layer instability, i.e., to the CF instability.

\section{CONCLUSIONS}

A detailed analysis of the properties of 3D boundary-layer disturbances evolving on a real airfoil section simulating a swept wing of infinite span has been carried out at an angle of attack of $-5^{\circ}$. In this case, the laminar boundary layer supports crossflow instabilities only, whereas the Tollmien-Schlichting instability is fully suppressed by a favorable streamwise pressure gradient. The experiments are performed at fully controlled disturbance conditions. The calculations of the base flow and the stability characteristics are carried out for particular experimental conditions.

It is shown that the unsteady perturbations excited in the sweptwing boundary layer in the experiment have properties of unsteady CF-instability modes developing in a linear way, i.e., independently of the disturbance amplitude.

All CF-instability characteristics of the $3 \mathrm{D}$ boundary layer developing on a real airfoil section (simulating an infinite-span swept wing) are investigated systematically in detail experimentally and compared with linear-stability theories. These characteristics include (i) disturbance eigenfunctions (amplitudes and phases), (ii) amplification curves, and (iii) a full set of dispersion characteristics (streamwise wavenumbers, phase speeds, and wave propagation angles) vs spanwise wavenumber and frequency. A very good agreement between all measured and calculated stability characteristics is obtained in a broad range of parameters for unsteady normal CF-instability modes including cases of quasistationary CF modes, which correspond physically to stationary CF vortices. According to our knowledge, such agreement had never been achieved previously for unsteady CF modes, in 3D boundary layers, especially not on real airfoils.

The next paper (the second part of the present study) will be devoted to a detailed investigation of the 3D Tollmien-Schlichting instability of a 3D boundary layer developing on the same airfoil section but installed at an angle of attack of $+1.5^{\circ}$ when the laminar-turbulent transition is dominated by the TS-instability mechanism.

\section{ACKNOWLEDGMENTS}

This work was supported by the European Commission through FP7 project RECEPT (Grant Agreement No. ACPO-GA2010-265094) and carried out (partly) within the framework of the Program of Fundamental Scientific Research of the Russian state academies of sciences in 2013-2020 (Project No. AAAA-A17117030610128-8). The experimental study includes a significant contribution of P. H. Alfredsson, N. Tillmark, and A. Segalini from KTH Stockholm.

\section{REFERENCES}

${ }^{1}$ P. Hall, M. R. Malik, and D. I. A. Poll, "On the stability of an infinite swept attachment line boundary layer,” Proc. R. Soc. A 395, 229 (1984).

${ }^{2}$ W. E. Gray, The Effect of Wing Sweep on Laminar Flow (Royal Aircraft Establishment, 1952), Vol. 227.

${ }^{3}$ U. Dallmann and H. Bieler, "Analysis and simplified prediction of primary instability of three-dimensional boundary-layer flows," in 19th AIAA, Fluid Dynamics, Plasma Dynamics, and Lasers Conference (American Institute of Aeronautics and Astronautics, 1987)

${ }^{4}$ M. V. Ustinov, "Laminar-turbulent transition in boundary layers (review). Part. 1: Main types of laminar-turbulent transition in a swept-wing boundary layer," TsAGI Sci. J. 44, 1 (2013).

${ }^{5}$ A. Dovgal, V. Kozlov, and A. Michalke, "Laminar boundary layer separation: Instability and associated phenomena," Prog. Aeronaut. Sci. 30, 61 (1994).

${ }^{6}$ V. I. Borodulin, A. V. Ivanov, and Y. S. Kachanov, "Swept-wing boundary-layer transition at various external perturbations: Scenarios, criteria, and problems of prediction," Phys. Fluids 29, 094101 (2017).

${ }^{7}$ H. Bippes, "Basic experiments on transition in three-dimensional boundary layers dominated by crossflow instability," Prog. Aerosp. Sci. 35, 363 (1999).

${ }^{8}$ V. R. Gaponenko, A. V. Ivanov, Y. S. Kachanov, and J. D. Crouch, "Swept-wing boundary-layer receptivity to surface non-uniformities," J. Fluid Mech. 461, 93 (2002).

${ }^{9}$ W. S. Saric, H. L. Reed, and E. B. White, "Stability and transition of threedimensional boundary layers," Annu. Rev. Fluid Mech. 35, 413 (2003).

${ }^{10}$ V. I. Borodulin, A. V. Ivanov, Y. S. Kachanov, and A. P. Roschektaev, "Receptivity coefficients at excitation of cross-flow waves by free-stream vortices in the presence of surface roughness," J. Fluid Mech. 716, 487 (2013).

${ }^{11}$ H. B. E. Kurz and M. J. Kloker, "Receptivity of a swept-wing boundary layer to micron-sized discrete roughness elements," J. Fluid Mech. 755, 62 (2014).

${ }^{12}$ R. Michel, D. Arnal, E. Coustols, and J. C. Juillen, "Experimental and theoretical studies of boundary layer transition on a swept infinite wing," in LaminarTurbulent Transition, edited by V. V. Kozlov (Springer Berlin Heidelberg, 1985), pp. 553-561.

${ }^{13}$ W. S. Saric and L. G. Yeates, "Generation of crossflow vortices in a threedimensional flat-plate flow," in Laminar-Turbulent Transition, edited by V. V. Kozlov (Springer Berlin Heidelberg, 1985), pp. 429-437.

${ }^{14}$ Y. Kohama, "Some expectation on the mechanism of cross-flow instability in a swept wing flow," Acta Mech. 66, 21 (1987).

${ }^{15}$ V. N. Zhigulyov and A. M. Tumin, Origin of Turbulence: Dynamic Theory of the Excitation and Evolution of Instabilities in Boundary Layers (Novosibirsk Izdatel Nauka, 1987).

${ }^{16}$ P. Nitschke-Kowsky and H. Bippes, "Instability and transition of a threedimensional boundary layer on a swept flat plate," Phys. Fluids 31, 786 (1988).

${ }^{17}$ Y. Kachanov, O. Tararykin, and A. Fedorov, "Experimental simulation of sweptwing boundary layer in a region of cross-flow formation," Izv. Sib. Otd. Akad. Nauk SSSR, Ser. Tekh. Nauk 3, 44 (1989).

${ }^{18}$ H. L. Reed and W. S. Saric, "Stability of three-dimensional boundary layers," Annu. Rev. Fluid Mech. 21, 235 (1989).

${ }^{19}$ Y. Kachanov, O. Tararykin, and A. Fedorov, "Investigation of stability of boundary layer on a model of a swept wing to stationary disturbances," Izv. Sib. Otd. Akad. Nauk SSSR, Ser. Tekh. Nauk 5, 11 (1990).

${ }^{20} \mathrm{H}$. Bippes and B. Müller, "Disturbance growth in an unstable threedimensional boundary layer," in Numerical and Physical Aspects of Aerodynamic Flows IV, edited by T. Cebeci (Springer Berlin Heidelberg, 1990), pp. 345-358. 
${ }^{21}$ D. Arnal, G. Casalis, and J. C. Juillen, "Experimental and theoretical analysis of natural transition on 'infinite' swept wing," in Laminar-Turbulent Transition, edited by D. Arnal and R. Michel (Springer Berlin Heidelberg, 1990), pp. 311-325.

${ }^{22}$ W. S. Saric, "Low-speed boundary-layer transition experiments," in Transition: Experiments, Theory and Computations, edited by T. Corke, G. Erlebacher, and M. Hussaini (Oxford University Press, New York, 1994), pp. 1-114.

${ }^{23}$ Y. S. Kachanov, "Generation, development and interaction of instability modes in swept-wing boundary layer," in IUTAM Symposium on Nonlinear Instability and Transition in Three-Dimensional Boundary Layers, edited by P. W. Duck and P. Hall (Springer Netherlands, Dordrecht, 1996), pp. 115-132.

${ }^{24}$ L. Hunt and W. Saric, "Boundary-layer receptivity of three-dimensional roughness arrays on a swept-wing," AIAA Paper 2011-3881, 2011.

${ }^{25}$ W. S. Saric, D. E. West, M. W. Tufts, and H. L. Reed, "Flight test experiments on discrete roughness element technology for laminar flow control," AIAA Paper 2015-0539, 2015.

${ }^{26} \mathrm{R}$. Radzetsky, Jr., M. Reibert, and W. Saric, "Development of a stationary crossflow vortices on a swept wing," in Fluid Dynamics Conference (American Institute of Aeronautics and Astronautics, 1994).

${ }^{27}$ L. M. Mack, "Boundary layer linear stability theory," AGARD Report No. 709, 1984.

${ }^{28}$ F. Meyer and L. Kleiser, "Numerical simulation of transition due to crossflow instability," in Laminar-Turbulent Transition, edited by D. Arnal and R. Michel (Springer Berlin Heidelberg, 1990), pp. 609-619.

${ }^{29} \mathrm{~N}$. Itoh, "Cross-flow instability of 3-D boundary layers on a flat plate," in Laminar-Turbulent Transition, edited by D. Arnal and R. Michel (Springer Berlin Heidelberg, 1990), pp. 359-368.

${ }^{30} \mathrm{~S}$. Takagi and N. Itoh, "Observation of traveling waves in the three-dimensional boundary layer along a yawed cylinder," Fluid Dyn. Res. 14, 167 (1994).

${ }^{31} \mathrm{H}$. Deyhle and H. Bippes, "Disturbance growth in an unstable threedimensional boundary layer and its dependence on environmental conditions," J. Fluid Mech. 316, 73 (1996).

${ }^{32}$ Y. S. Kachanov and O. I. Tararykin, "The experimental investigation of stability and receptivity of a swept-wing flow," in Laminar-Turbulent Transition, edited by D. Arnal and R. Michel (Springer Berlin Heidelberg, 1990), pp. $499-509$.

${ }^{33}$ M. Reibert, W. Saric, R. Carrillo, Jr., and K. Chapman, "Experiments in nonlinear saturation of stationary crossflow vortices in a swept-wing boundary layer," in 34th Aerospace Sciences Meeting and Exhibit (American Institute of Aeronautics and Astronautics, 1996).

${ }^{34}$ T. S. Haynes and H. L. Reed, "Numerical simulation of swept-wing vortices using nonlinear parabolized stability equations," in General, Corporate and Regional Aviation Meeting and Exposition (SAE International, 1997).

${ }^{35}$ J. Dagenhart, W. Saric, M. Mousseux, and J. Stack, "Crossflow-vortex instability and transition on a 45 deg swept wing," in 20th Fluid Dynamics, Plasma Dynamics and Lasers Conference (American Institute of Aeronautics and Astronautics, 1989).

${ }^{36}$ Y. Kachanov and O. Tararykin, "Interaction between the stationary and traveling instability wave on a swept wing," in Methods of Aerophysical Research, edited by A. Kharitonov (Institute of Theoretical and Applied Mechanics, Novosibirsk, 1990), pp. 138-143.

${ }^{37}$ J. R. Dagenhart, W. S. Saric, J. A. Hoos, and M. C. Mousseux, "Experiments on swept-wing boundary layers," in Laminar-Turbulent Transition, edited by D. Arnal and R. Michel (Springer Berlin Heidelberg, 1990), pp. 369-380.

${ }^{38} \mathrm{~B}$. Müller, "Experimental study of the travelling waves in a three-dimensional boundary layer," in Laminar-Turbulent Transition, edited by D. Arnal and R. Michel (Springer Berlin Heidelberg, 1990), pp. 489-498.

${ }^{39} \mathrm{H}$. Deyhle, G. Hoehler, and H. Bippes, "Experimental investigation of instability wave propagation in a three-dimensional boundary-layer flow," AIAA J. 31, 637 (1993).

${ }^{40} \mathrm{H}$. Bippes, "Instability features appearing on swept wing configurations," in Laminar-Turbulent Transition, edited by D. Arnal and R. Michel (Springer Berlin Heidelberg, 1990), pp. 419-430.

${ }^{41}$ J. D. Crouch, V. R. Gaponenko, A. V. Ivanov, and Y. S. Kachanov, "Theoretical and experimental comparisons of the stability and receptivity of swept-wing boundary layers," in Division of Fluid Dynamics Meeting Abstracts (American Physical Society, 1997), p. Dc.04.

${ }^{42}$ V. I. Borodulin, V. R. Gaponenko, A. V. Ivanov, Y. S. Kachanov, and J. D. Crouch, "Swept-wing boundary-layer stability to stationary and traveling disturbances. Experiment and theory," in Stability of Homogeneous and Heterogeneous Liquid Flows, edited by V. Y. Rudyak (NGASU, Novosibirsk, 2000), pp. 150-153.

${ }^{43}$ M. R. Malik, F. Li, and C.-L. Chang, "Crossflow disturbances in threedimensional boundary layers: Nonlinear development, wave interaction and secondary instability," J. Fluid Mech. 268, 1-36 (1994).

${ }^{44}$ F. P. Bertolotti, "A partial simulation of receptivity and transition in 3-D boundary layers," in Laminar-Turbulent Transition, edited by R. Kobayashi (Springer Berlin Heidelberg, 1995), pp. 491-498.

${ }^{45}$ S. Hein, A. Hanifi, and G. Casalis, "Nonlinear transition prediction," in European Congress on Computational Methods in Applied Sciences and Engineering ECCOMAS 2000 Barcelona, 11-14 September 2000.

${ }^{46} \mathrm{~S}$. Hein, "Linear and nonlinear growth of secondary instabilities of stationary crossflow vortices studied by parabolized stability equations," in New Results in Numerical and Experimental Fluid Mechanics XI, Notes on Numerical Fluid Mechanics and Multidisciplinary Design, Contributions to the 20th STAB/DGLR Symposium Braunschweig, Germany 2016, edited by A. Dillmann et al. (Springer, 2018), Vol. 136, pp. 219-228.

${ }^{47} \mathrm{M}$. Högberg and D. Henningson, "Secondary instability of cross-flow vortices in Falkner-Skan-Cooke boundary layers," J. Fluid Mech. 368, 339 (1998).

${ }^{48} \mathrm{P}$. Wassermann and M. Kloker, "Mechanisms and passive control of crossflowvortex-induced transition in a three-dimensional boundary layer," J. Fluid Mech. 456, 49 (2002).

${ }^{49} \mathrm{P}$. Wassermann and M. Kloker, "Transition mechanisms induced by travelling crossflow vortices in a three-dimensional boundary layer," J. Fluid Mech. 483, 67 (2003).

${ }^{50}$ D. Tempelmann, A. Hanifi, and D. S. Henningson, "Swept-wing boundary-layer receptivity," J. Fluid Mech. 700, 490 (2012).

${ }^{51}$ D. Tempelmann, L.-U. Schrader, A. Hanifi, L. Brandt, and D. S. Henningson, "Swept wing boundary-layer receptivity to localised surface roughness," J. Fluid Mech. 711, 516 (2012).

${ }^{52}$ S. M. Hosseini, D. Tempelmann, A. Hanifi, and D. S. Henningson, "Stabilization of a swept-wing boundary layer by distributed roughness elements," J. Fluid Mech. 718, R1 (2013).

${ }^{53}$ M. Brynjell-Rahkola, N. Shahriari, P. Schlatter, A. Hanifi, and D. S. Henningson, "Stability and sensitivity of a cross-flow-dominated falknerskancooke boundary layer with discrete surface roughness," J. Fluid Mech. 826, 830-850 (2017).

${ }^{54}$ V. I. Borodulin, A. V. Ivanov, Y. S. Kachanov, R. Örlü, A. Hanifi, and S. Hein, "Characteristics of 3D instability of a 35-degree swept wing to CF and TS modes. Experiment and theory," AIP Conf. Proc. 1770, 030054 (2016).

${ }^{55}$ D. Romano, P. Alfredsson, A. Hanifi, R. Örlü, N. Tillmark, V. Borodulin, A. Ivanov, Y. Kachanov, and M. Minervino, "Design and tests of wind-tunnel sidewalls for receptivity experiments on a swept wing," in Mechanical and Aerospace Engineering IV, Applied Mechanics and Materials (Trans Tech Publications, 2013), Vol. 390, pp. 96-102.

${ }^{56}$ A. Hanifi, S. Hein, D. Romano, M. Minervino, W. Würz, V. Borodulin, A. Ivanov, and Y. Kachanov, "Numerical and experimental realization of an infinite-swept-wing boundary-layer flow in a wind tunnel," in Proceedings of 17th International Conference on Methods of Aerophysical Research, edited by V. Fomin (ITAM SB RAS, Novosibirsk, 2014).

${ }^{57}$ V. Borodulin, A. Ivanov, and Y. Kachanov, "Numerical and experimental realization of an infinite-swept-wing boundary-layer flow in a wind tunnel," in Proceedings of 17th International Conference on Methods of Aerophysical Research, edited by V. Fomin (ITAM SB RAS, Novosibirsk, 2014).

${ }^{58}$ Y. S. Kachanov, "Development of spatial wave packets in boundary layer," in Laminar-Turbulent Transition, edited by V. V. Kozlov (Springer Berlin Heidelberg, 1985), pp. 115-123.

${ }^{59}$ V. R. Gaponenko, A. V. Ivanov, and Y. S. Kachanov, "Experimental study of a swept-wing boundary-layer stability with respect to unsteady disturbances," Thermophys. Aeromech. 2, 287 (1995). 
${ }^{60}$ A. V. Boiko, A. V. Ivanov, Y. S. Kachanov, and D. A. Mischenko, "Steady and unsteady Görtler boundary-layer instability on concave wall," Eur. J. Mech.: B/Fluids 29, 61 (2010).

${ }^{61}$ A. Hanifi, D. Henningson, S. Hein, F. Bertolotti, and M. Simen, "Linear nonlocal instability analysis-the linear NOLOT code," Technical Report FFA TN 54, 1994.

${ }^{62}$ S. Hein, F. Bertolotti, M. Simen, A. Hanifi, and D. Henningson, "Linear nonlocal instability analysis-the linear NOLOT code," Technical Report DLR-IB 223-94 A43, 1994.
${ }^{63}$ A. Ivanov and Y. Kachanov, "Excitation and development of spatial packets of instability waves in a three-dimensional boundary layer," Thermophys. Aeromech. 1, 287 (1994).

${ }^{64}$ V. R. Gaponenko, A. V. Ivanov, and Y. S. Kachanov, "Experimental study of cross-flow instability of a swept-wing boundary layer with respect to traveling waves," in Laminar-Turbulent Transition, edited by R. Kobayashi (Springer Berlin Heidelberg, Berlin, Heidelberg, 1995), pp. 373-380. 